\title{
Microscopic design and optimization of hydrodynamically lubricated dissipative interfaces
}

\author{
Berkay Alp Çakal $^{1} \mid$ İlker Temizer $^{1}(\mathbb{D}) \mid$ Kenjiro Terada $^{2}(\mathbb{D}) \mid$ Junji Kato $^{3}$ (i)
}

${ }^{1}$ Department of Mechanical Engineering, Bilkent University, Ankara, Turkey

${ }^{2}$ International Research Institute of Disaster Science, Tohoku University, Sendai, Japan

${ }^{3}$ Department of Civil Engineering,

Nagoya University, Nagoya, Japan

\section{Correspondence}

İlker Temizer, Department of Mechanical Engineering, Bilkent University, 06800

Ankara, Turkey.

Email: temizer@bilkent.edu.tr

\begin{abstract}
Summary
A homogenization-based topology optimization framework is developed, which can endow hydrodynamically lubricated interfaces with a micro-texture, to achieve optimal macroscopic responses by addressing both dissipative and nondissipative physics at the interface. With respect to the homogenization aspects of the problem, the thermodynamic consistency of the two-scale formulation is explicitly analyzed and verified. With respect to the topology optimization aspects, a variational approach to sensitivity analysis is pursued. Subsequently, these are employed in micro-texture design studies, which address microscopic and macroscopic objectives. The influence of dissipation on the optimization results is demonstrated through extensive numerical investigations, which also highlight the importance of working in a sufficiently flexible design space that can deliver nearly optimal micro-texture geometries.
\end{abstract}

\section{KEYWORDS}

dissipation, homogenization, lubrication, optimization, texture design

\section{1 | INTRODUCTION}

The macroscopic mechanical behavior of an interface can be tailored by endowing it with a suitably designed micro-texture. The goal of such a multiscale engineering task typically entails the simultaneous consideration of multiple challenging and possibly conflicting demands on the design process. The major goal of this work is to address such a task in the context of hydrodynamically lubricated interfaces, where a homogenization-based two-scale analysis will be employed to efficiently and accurately link the interacting scales, and topology optimization is employed to obtain optimal micro-texture in a flexible design space.

The exposition in this study is based on two earlier works, ${ }^{1,2}$ where a combination of modern topology optimization ${ }^{3,4}$ and homogenization methods ${ }^{5,6}$ were pursued for the first time in the context of micro-texture design and optimization in hydrodynamic lubrication. Following the terminology in the work of Waseem et al, ${ }^{2}$ the scope of this study is focused on two classes of optimization problems. The first class is microscopic objective optimization (MICOO), where the goal is to tailor the mechanical behavior of a micro-texture without solving a macroscopic problem. Such a process is typically driven directly in terms of quantities which characterize the macroscopic constitutive response. In the context of material design, example quantities are Poisson's ratio and the thermal expansion coefficient, which may attain nonconventional values through suitable microstructure designs, ${ }^{7-9}$ or the elasticity tensor that may be required to match a prescribed target value. ${ }^{9-11}$ In the context of micro-texture design, similar optimization problems can be cast in terms of so-called flow factor tensors, which appear in the formulation of the macroscopic mechanics of hydrodynamic lubrication, and this particular goal has been the subject of research in the work of Waseem et al. ${ }^{1}$ The second class of problems is macroscopic objective optimization (MACOO), where the focus is on quantities of interest which require the solution of a macroscopic 
boundary value problem and, hence, is intrinsically driven by the two-scale analysis of homogenization. In structural analysis, minimizing deflection under a prescribed load is a very specific but a practically important example. ${ }^{12-15}$ The counterpart of such an optimization task in hydrodynamic lubrication is the design of a micro-texture which can maximize the load capacity of the interface, as recently investigated in the work of Waseem et al. ${ }^{2}$ All of these works presented and investigated homogenization-based topology optimization approaches, which is the distinguishing feature of the current study as well. Focusing on hydrodynamic lubrication in the broad field of tribology, there is a limited number of works that addresses microscopic interface design in comparison to material design. Note that the present focus is on the design of the interface and not on the analysis of a prescribed texture (see the work of Gropper et $\mathrm{al}^{16}$ for a review of commonly prescribed texture geometries and related analysis approaches). One advantage of existing design studies is their ability to address the problem directly through a complete mesoscale analysis in the lack of a clear scale separation that is necessary for the application of the homogenization theory. ${ }^{17-20}$ Another advantage is that, considering the large number of design variables that are typically needed in topology optimization, they can restrict the design space to achieve optimal designs in a numerically more efficient manner. ${ }^{21-23}$ On the other hand, an ability to endow interfaces with very finely distributed intricate geometries using modern manufacturing methods provides an opportunity to question and explore the possibilities of unrestricted microscopic interface geometry design via homogenization-based topology optimization. This is the underlying motivation of this study, where, specifically, the approaches developed in the works of Waseem et al ${ }^{1,2}$ are extended to incorporate dissipative effects at the interface as the primary novel contribution. In realizing this extension, a rigorous homogenization-based characterization of dissipation will be developed, the thermodynamic consistency of the theoretical framework will be established, the physical relevance of the numerical results will be critically examined through analytical estimates, and the role of the optimization domain geometry parameters as additional design variables will be highlighted, which together constitute the secondary novel contribution of this work.

Realizing the extension stated earlier requires a careful reconsideration of homogenization in hydrodynamically lubricated dissipative interfaces as well as its sensitivity analysis for topology optimization. Toward this purpose, the classical mechanical model of the hydrodynamic lubrication interface is first reviewed in Section 2, with the main purpose of clarifying the dissipative aspects of the problem. This review is set in a general setting where there is no restriction on the geometry or motion of the interacting surfaces. Such a general setting also provides a sound basis for a discussion on the role of various simplifications that are typically invoked in the literature in the analysis of this multiscale interface problem. On the other hand, it also requires a careful consideration of homogenization with a focus on dissipation, which is addressed in Section 3. The general setting is then narrowed down to a special yet, from a practical perspective, sufficiently broad setting in Section 4. The discussions in these sections are accompanied by a careful selection of references from the literature, which not only emphasizes past studies that form the basis of the current study but also helps highlight some additional novel aspects of the presented approach. In particular, the thermodynamic consistency of the two-scale formulation will be verified in Section 5 by exploiting the properties of the constitutive tensors that characterize the macroscopic interface response. Based on the developed formulation, Section 6 first discusses a variational analysis of the sensitivity of these tensors with respect to micro-texture design variables and then employs the results of this analysis to formulate the sensitivity of fundamental macroscopic objective functions, which may be employed to drive micro-texture topology optimization. The particular problem settings and numerical investigations based on these are then presented in Section 7, which are further examined in Section 8 with respect to optimality. The discussion is concluded by a summary and an outlook in Section 9 .

\section{2 | REYNOLDS EQUATION}

Multiscale analysis involving the Reynolds equation has been the subject of many studies, which, however, predominantly concentrate on nondissipative aspects such as the pressure distribution within the interface. Therefore, it is necessary to give a compact summary of the interface physics with a view toward providing a clear basis for dissipative effects, following largely standard expositions, ${ }^{24,25}$ but by omitting cavitation and contact. Note that the classification of dissipative versus nondissipative is mainly with respect to the macroscopically observed response of the interface and is introduced here to emphasize the focus of this work. Although hydrodynamic lubrication is necessarily accompanied by microscopic dissipation in the interface fluid, the generated pressure may not be directly linked with the total power input from the macroscale that is necessary to sustain the lubrication mechanism or it may be only partially responsible for it. In such cases, a complete description of the heating at the interface requires the additional consideration of macroscopic tangential tractions that embody all or remaining microscopic dissipation effects. 
The Reynolds equation expresses the effective response of two interacting physical surfaces $\mathcal{P}^{ \pm}$in close proximity (Figure 1). Its classical derivation assumes an incompressible Newtonian fluid with viscosity $\mu$ in the absence of inertia as well as an interface geometry which induces a slowly varying and vanishingly small fluid film thickness (see also the work of Bayada and Chambat ${ }^{26}$ for a derivation based on Stokes equations). The Reynolds equation is essentially expressed on a lubrication surface $\mathcal{L}$, which, in principle, can be independent from either of the two physical surfaces ${ }^{27}$ but is typically taken to be coincident with one of them. For the types of problems to be addressed in this study, it is sufficient to consider $\mathcal{L}$ to be a flat and stationary surface with an in-plane position vector $\boldsymbol{x}=x_{i} \boldsymbol{e}_{i}$, where $\boldsymbol{e}_{i}(i=1$ or 2$)$ are tangential unit vectors, and an out-of-plane position $n$ in the normal direction $v$ to $\mathcal{L}$, pointing from $\mathcal{P}^{-}$to $\mathcal{P}^{+}$. Subsequently, denoting the out-of-plane position of the upper/lower physical surface $\mathcal{P}^{ \pm}$by $n^{ \pm}$, the film thickness at any point on $\mathcal{L}$ may be expressed as

$$
h(\boldsymbol{x}, t)=n^{+}(\boldsymbol{x}, t)-n^{-}(\boldsymbol{x}, t),
$$

where a dependence on time $(t)$ is also included. The film thickness is not well-defined in the presence of re-entrant features on the surfaces, although a definition based on a simple modification of the surfaces may still enable one to proceed with the following analysis in the context of homogenization. ${ }^{28}$ The mechanics of lubrication is possibly active only over a subdomain of $\mathcal{L}$, but this will not be explicitly indicated. Within the Reynolds equation approximation, the tangential velocity variation $\widetilde{\boldsymbol{U}}(n)$ of the fluid in the normal direction is quadratic and defines the fluid flux at any point on $\mathcal{L}$ via

$$
\boldsymbol{q}=\int_{n^{-}}^{n^{+}} \tilde{\boldsymbol{U}} \mathrm{d} n
$$

The value of $\tilde{\boldsymbol{U}}$ on the upper/lower surface will be denoted by $\boldsymbol{U}^{ \pm}=U_{i}^{ \pm} \boldsymbol{e}_{i}$ and the surface velocity components along $\boldsymbol{v}$ will be denoted by $W^{ \pm}$. These velocities do not depend on position or time in the present setting. The film thickness rate of change can now be locally evaluated via

$$
\frac{\partial h}{\partial t}=\left(W^{+}-W^{-}\right)-\left(\nabla n^{+} \cdot \boldsymbol{U}^{+}-\nabla n^{-} \cdot \boldsymbol{U}^{-}\right)
$$

The sum and difference of the tangential velocities will be indicated by

$$
\boldsymbol{U}=\boldsymbol{U}^{+}+\boldsymbol{U}^{-}, \quad \boldsymbol{V}=\boldsymbol{U}^{+}-\boldsymbol{U}^{-} .
$$

The relative motion of the surfaces, together with the boundary conditions on the interface geometry, generates a pressure $p$ with gradient $\boldsymbol{g}=\nabla p$ at the interface that is governed by the Reynolds equation

$$
-\nabla \cdot \boldsymbol{q}=\frac{\partial h}{\partial t},
$$

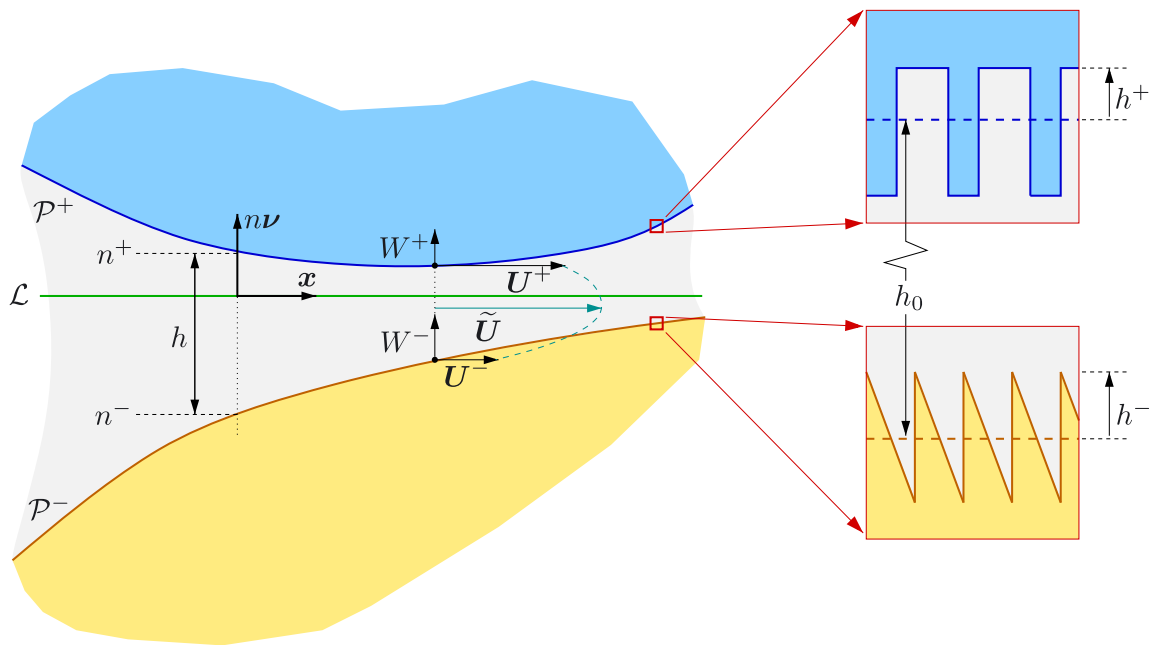

FIGURE 1 The geometry of the micro-textured lubrication interface [Colour figure can be viewed at wileyonlinelibrary.com] 
where the fluid flux $\boldsymbol{q}$ has a combined Poiseuille-Couette constitutive form that is obtained from (2), ie,

$$
\boldsymbol{q}=-a \boldsymbol{g}+b \boldsymbol{U}
$$

Here, the following coefficients have been defined, together with $e$ for future reference:

$$
a=\frac{h^{3}}{12 \mu} \quad, \quad b=\frac{h}{2} \quad, \quad e=\frac{\mu}{h} .
$$

Note that, despite the original assumption of a smoothly varying film thickness in the derivation of the Reynolds equation, the theory retains its predictive capability in the presence of sharp transitions as well with respect to a more general framework based on the Stokes equations in the context of homogenization. ${ }^{28}$ Here, a sharp transition refers to a steep slope in the film thickness within a localized region of the cell and, in the extreme case, could indicate a jump. Indeed, large but isolated step changes in the film thickness are also classically attacked based on the Reynolds equation. ${ }^{24,25}$ It is emphasized that the physical dimensions of the micro-texture within which the film thickness variation occurs will be an additional and independent factor (see Section 3.2).

In order to equilibriate the resulting tractions applied to $\mathcal{P}^{ \pm}$by the fluid in the normal direction, tractions $\mp p v$ must be externally applied to $\mathcal{P}^{ \pm}$. Indicating the equilibriating tractions in the tangential direction with $\boldsymbol{\tau}^{ \pm}=\tau_{i}^{ \pm} \boldsymbol{e}_{i}$, the total equilibriating traction applied to each surface may be expressed as

$$
\boldsymbol{t}^{ \pm}=\mp p \boldsymbol{\nu}+\tau^{ \pm}
$$

Alternatively stated, $-\boldsymbol{t}^{ \pm}$would act as boundary conditions on $\mathcal{P}^{ \pm}$in the solution of an elastohydrodynamic problem. The tangential tractions may be decomposed as

$$
\tau^{ \pm}=\tau_{p}^{ \pm}+\tau_{v}^{ \pm},
$$

where the first term accounts for the contribution by the pressure in the tangential direction via

$$
\tau_{p}^{ \pm}= \pm p \nabla n^{ \pm}
$$

and the second term accounts for the shear tractions, which are generated due to viscous flow:

$$
\tau_{v}^{ \pm}=b \boldsymbol{g} \pm e \boldsymbol{V}
$$

These tangential tractions are balanced by the normal tractions acting on the boundary $\partial \mathcal{L}$ with outward unit normal $\boldsymbol{m}$, which is a statement of equilibrium for the interface, ie,

$$
0=\int_{\mathcal{L}}\left(\boldsymbol{\tau}^{+}+\boldsymbol{\tau}^{-}\right) \mathrm{d} a+\int_{\partial \mathcal{L}}(-p \boldsymbol{m}) h \mathrm{~d} l
$$

Based on the foregoing expressions and standard manipulations, one can also verify that the total stress power $\pi \geq 0$ within the fluid, subject to the simplifications on the stress tensor and the velocity field in the context of the Reynolds equation, is equal to the rate of work done by the tractions, ie,

$$
\pi=\int_{\partial \mathcal{L}}(-p) \boldsymbol{q} \cdot \boldsymbol{m} \mathrm{d} l+\int_{\mathcal{L}}\left(\boldsymbol{\tau}^{+} \cdot \boldsymbol{U}^{+}+\boldsymbol{\tau}^{-} \cdot \boldsymbol{U}^{-}\right) \mathrm{d} a+\int_{\mathcal{L}}(-p)\left(W^{+}-W^{-}\right) \mathrm{d} a \geq 0 .
$$

The stress power is purely dissipative and hence contributes to the heating of the interface. ${ }^{29,30}$ In many cases, the dissipative effects are associated with a friction coefficient. ${ }^{24,25}$ In this work, the dissipation will be monitored directly instead. 


\section{3 | HOMOGENIZATION ANALYSIS}

\section{1 | Reynolds equation}

The homogenization of the Reynolds equation delivers all microscopic fields, which are central to the construction of the flow factor tensors that help define the macroscopic interface response. In order to elucidate the particular forms of these tensors which will be employed in the upcoming sections as well as their relation to macroscopic expressions that predate the homogenization theory, it is advantageous to provide a compact summary of the homogenization process in a form that addresses the most general setting, where both of the interacting surfaces could be rough and moving. Although this process derives its basis from the first applications of the homogenization theory to hydrodynamic lubrication, ${ }^{31}$ the approach that is suitable to the general setting was first discussed in the work of Bayada et al. ${ }^{32}$ Starting with this section, this latter formulation will be outlined, with an additional step where a flow factor tensor will be introduced not only for the Poiseuille term but also for the Couette term, as in averaging-based methods. ${ }^{33,34}$ Overall, the aim is two-fold: (1) to present the homogenized form of the dissipation and the accompanying tractions, and (2) to build a basis for the microscopic sensitivity analysis of dissipation for the purposes of micro-texture topology optimization.

The formulation summarized in the previous section directly applies to a heterogeneous interface. In order to analyze this setting, it is sufficient to replace $\{\boldsymbol{x}, t\}$ with $\left\{\boldsymbol{x}_{\varepsilon}, t_{\varepsilon}\right\}$, where $\varepsilon$ is the ratio of representative in-plane microscopic and macroscopic dimensions, such as the micro-texture period to the interface length. Subsequently, operators $\left\{\nabla, \frac{\partial}{\partial t}\right\}$ and variables that depend on $\{\boldsymbol{x}, t\}$ are now admitted to depend on $\left\{\boldsymbol{x}_{\varepsilon}, t_{\varepsilon}\right\}$. For the variables, this dependence will be shown compactly, through a subscript $\varepsilon$. Moreover, since $\varepsilon \rightarrow 0$ in the limit of scale separation, the following decomposition is convenient:

$$
\boldsymbol{x}_{\varepsilon}=\boldsymbol{x}+\varepsilon \boldsymbol{y}, \quad t_{\varepsilon}=t+\varepsilon \tau,
$$

where $\boldsymbol{x}$ and $t$ will now refer to macroscopic position and time, whereas $\boldsymbol{y}$ and $\tau$ indicate microscopic position and time within a periodic cell $\mathcal{Y}$ and a motion period $\mathcal{T}$, respectively. Consequently, the operators take the explicit forms

$$
\nabla_{\boldsymbol{x}_{\varepsilon}}=\nabla_{\boldsymbol{x}}+\varepsilon^{-1} \nabla_{\boldsymbol{y}}, \quad \frac{\partial}{\partial t_{\varepsilon}}=\frac{\partial}{\partial t}+\varepsilon^{-1} \frac{\partial}{\partial \tau} .
$$

Additionally, for space-averaging over $\mathcal{Y}$, the notation $\langle\cdot\rangle=\frac{1}{|\mathcal{Y}|} \int_{\mathcal{Y}} \cdot \mathrm{d} a$ will be employed. The film thickness variation

$$
h_{\varepsilon}=n_{\varepsilon}^{+}-n_{\varepsilon}^{-}
$$

will now be simplified by the explicit forms

$$
n_{\varepsilon}^{ \pm}=n_{0}^{ \pm}(\boldsymbol{x}, t)+h^{ \pm}\left(\boldsymbol{y}-\tau \boldsymbol{U}^{ \pm}\right)
$$

where $\left\langle h^{ \pm}\right\rangle=0$ is assumed (Figure 1). This form explicitly invokes that the periodic $h^{ \pm}$variations, which are due to the micro-textures on $\mathcal{P}^{ \pm}$, do not display macroscopic variations. One may then also write

$$
h_{0}=n_{0}^{+}-n_{0}^{-} \quad \longrightarrow \quad h_{\varepsilon}=h_{0}+h^{+}-h^{-},
$$

where $h_{0}$ represents the local mean film thickness. In analogy with earlier definitions, the following definitions are useful:

$$
a_{0}=\frac{h_{0}^{3}}{12 \mu} \quad, \quad b_{0}=\frac{h_{0}}{2} \quad, \quad \bar{b}=\frac{1}{2}\left(h^{+}+h^{-}\right) \quad, \quad e_{0}=\frac{\mu}{h_{0}} .
$$

In order to obtain the homogenized response, the asymptotic expansion

$$
p_{\varepsilon}=p_{0}+\varepsilon p_{1}+\varepsilon^{2} p_{2}+\ldots
$$

is substituted into the Reynolds equation. Defining $\boldsymbol{G}=\nabla_{\boldsymbol{x}} p_{0}$, the linear expansion

$$
p_{1}=\boldsymbol{\omega} \cdot \boldsymbol{G}-\boldsymbol{\Omega} \cdot \boldsymbol{V}
$$


is introduced, where $\{\boldsymbol{\omega}, \boldsymbol{\Omega}\}$ are periodic vector fields, which embody the physical influence of the micro-texture on the macroscopic interface response and depend on $\{\boldsymbol{x}, \boldsymbol{y}, t, \tau\}$ with gradients

$$
\lambda=\nabla_{\boldsymbol{y}} \boldsymbol{\omega} \rightarrow \lambda_{j}^{i}=\frac{\partial \omega_{i}}{\partial y_{j}} \quad, \quad \boldsymbol{\Lambda}=\nabla_{\boldsymbol{y}} \boldsymbol{\Omega} \rightarrow \Lambda_{j}^{i}=\frac{\partial \Omega_{i}}{\partial y_{j}}
$$

Here, the components are explicitly shown due to the special index notation employed for these tensors. Omitting the details, the procedure delivers the following results when $\varepsilon \rightarrow 0$ is invoked. First, one finds that $p_{0}=p_{0}(\boldsymbol{x}, t, \tau)$, ie, $p_{0}$ does not depend on microscopic position although a dependence on microscopic time is retained. Second, microscopic cell problems are obtained for vectors $\{\boldsymbol{\omega}, \boldsymbol{\Omega}\}$. In weak form, these problems read

$$
\left\langle(a \boldsymbol{I}+a \lambda) \nabla_{\boldsymbol{y}} \phi\right\rangle=0 \quad, \quad\left\langle(\bar{b} \boldsymbol{I}+a \boldsymbol{\Lambda}) \nabla_{\boldsymbol{y}} \Phi\right\rangle=0,
$$

where $\{\phi, \Phi\}$ are periodic test functions and $\boldsymbol{I}$ is the identity tensor. Here, coefficents such as $a$ are defined through $h_{\varepsilon}$ but an explicit subscript $\varepsilon$ is not attached for a simpler notation. Moreover, note that (20) implies $\lim _{\varepsilon \rightarrow 0}\left\langle\boldsymbol{g}_{\varepsilon}\right\rangle=\boldsymbol{G}$. Now, upon solving the problems, the macroscopic flux $\boldsymbol{Q}(\boldsymbol{x}, t, \tau)=\lim _{\varepsilon \rightarrow 0}\left\langle\boldsymbol{q}_{\varepsilon}\right\rangle$ may be expressed as, with $(\cdot)^{T}$ indicating transpose,

$$
\boldsymbol{Q}=-\left\langle a \boldsymbol{I}+a \lambda^{T}\right\rangle \boldsymbol{G}+b_{0} \boldsymbol{U}+\left\langle a \boldsymbol{\Lambda}^{T}\right\rangle \boldsymbol{V}
$$

which satisfies the macroscopic Reynolds equation

$$
-\nabla_{\boldsymbol{x}} \cdot \boldsymbol{Q}=\frac{\partial h_{0}}{\partial t}
$$

The space-averaged tensorial quantities appearing within $\boldsymbol{Q}$ will be called flow factor tensors, following the terminology of Tripp ${ }^{33}$ that is based on the original proposal by Patir and Cheng. ${ }^{35}$ Explicit definitions, which will be useful for the purposes of the present study, will be stated in Section 4. Note that the solutions of the cell problems are unique up to a constant. A unique solution can be imposed via the classical conditions $\langle\boldsymbol{\omega}\rangle=\mathbf{0}$ and $\langle\Omega\rangle=\mathbf{0}$, or simply by prescribing the values of these variables at a chosen point of the cell.

\section{2 | Dissipation}

Note that a vanishing micro-texture period, ie, $\varepsilon \rightarrow 0$, was invoked after employing the Reynolds equation on the microscale. Hence, the physical value of the period does not matter as long as it is sufficiently small. If the starting point is the more general Stokes equations, then the macroscopic response is influenced by the particular physical value. This has first been highlighted in the work of Elrod ${ }^{36}$ and numerically first demonstrated in another work of Elrod ${ }^{37}$ and subsequently in the work of Mitsuya and Fukui. ${ }^{38}$ A rigorous mathematical basis for this influence was provided through homogenization theory in the work of Bayada and Chambat, ${ }^{31}$ which also clarified how to efficiently formulate the macroscopic response for all physical values of the period, which was numerically demonstrated only recently in the works of Ylldiran et $\mathrm{al}^{28}$ and Fabricius et al. ${ }^{39}$ Based on these studies, one may state that a physical realization of the micro-textures designed in the present study by invoking the Reynolds equation on the microscale must be such that the micro-texture period is small enough for homogenization to be meaningful but not too small to avoid violating the assumptions of the Reynolds equation, ie, the interface geometry must fall in the Reynolds regime. ${ }^{36}$ In this context, it is clear that the macroscopic characterization of the dissipation will also depend on the physical value of the period if the starting point is the Stokes equations, as indeed demonstrated in the work of Mitsuya and Fukui. ${ }^{38}$ A homogenization approach similar to the work of Bayada and Chambat, ${ }^{31}$ which clarifies an efficient macroscopic formulation of the tangential tractions for different period values, was carried out comparatively recently. ${ }^{40}$ It is highlighted that the homogenized traction expressions summarized later agree with the expressions stated therein for the Reynolds regime in the unilateral setting that will be discussed in Section 4 after some straightforward manipulations. For a comparison of the expressions, see Theorem 3.4 ( $\alpha>3 / 2$ case) for the rough/stationary surface and Theorem 3.5 ( $\alpha>1$ case) for the smooth/moving surface. 
Making use of (8), the macroscopic tractions are

$$
\boldsymbol{T}^{ \pm}=\lim _{\varepsilon \rightarrow 0}\left\langle\boldsymbol{t}_{\varepsilon}^{ \pm}\right\rangle=\mp p_{0} \boldsymbol{\nu}+\mathcal{T}^{ \pm}
$$

Here, $\mathcal{T}^{ \pm}$and its individual components are similarly defined through averaging, for example $\mathcal{T}^{ \pm}=\lim _{\varepsilon \rightarrow 0}\left\langle\boldsymbol{\tau}_{\varepsilon}^{ \pm}\right\rangle$. Omitting the details, straightforward manipulations, which particularly make use of the periodicity of the microscopic quantities, deliver

$$
\boldsymbol{\mathcal { T }}_{p}^{ \pm}= \pm p_{0} \nabla_{\boldsymbol{x}} n_{0}^{ \pm} \mp\left\langle h^{ \pm} \lambda^{T}\right\rangle \boldsymbol{G} \pm\left\langle h^{ \pm} \boldsymbol{\Lambda}^{T}\right\rangle \boldsymbol{V}
$$

as well as

$$
\mathcal{T}_{v}^{ \pm}=\left\langle b \boldsymbol{I}+b \lambda^{T}\right\rangle \boldsymbol{G} \pm\left\langle e \boldsymbol{I} \mp b \boldsymbol{\Lambda}^{T}\right\rangle \boldsymbol{V} .
$$

The space-averaged tensorial quantities appearing within the traction expressions will be called stress factor tensors, following the choice of Tripp ${ }^{33}$ in combination with the original proposal by Patir and Cheng. ${ }^{41}$ Explicit definitions will be stated in Section 4. Finally, the macroscopic interface dissipation $\Pi=\lim _{\varepsilon \rightarrow 0}\left\langle\pi_{\varepsilon}\right\rangle \geq 0$ may be stated as

$$
\Pi=\int_{\partial \mathcal{L}}\left(-p_{0}\right) \boldsymbol{Q} \cdot \boldsymbol{m} \mathrm{d} l+\int_{\mathcal{L}}\left(\mathcal{T}^{+} \cdot \boldsymbol{U}^{+}+\mathcal{T}^{-} \cdot \boldsymbol{U}^{-}\right) \mathrm{d} a+\int_{\mathcal{L}}\left(-p_{0}\right)\left(W^{+}-W^{-}\right) \mathrm{d} a \geq 0 .
$$

Note that the inequality is stated as an expectation at this stage, based on the fact that the macroscopic dissipation is an average of its microscopic counterpart. However, the explicit representation of $\Pi$ via macroscopic quantities requires an independent verification of this inequality to ensure the thermodynamic consistency of the two-scale formulation, which will be the subject of Section 5 .

At this point, it is useful to review earlier work on dissipative effects in the context of multiscale hydrodynamic lubrication, without an attempt to provide an exhaustive coverage. Current formulations based on stress factors originate from the study in the work of Patir and Cheng, ${ }^{41}$ where the shear stresses were first derived through an averaging-based method by assuming macroscopic isotropy or simple cases of anisotropy. Therein, the contributions related to not only $\mathcal{T}_{v}^{ \pm}$but also to $\mathcal{T}_{p}^{ \pm}$were already highlighted. Their approach was based on earlier work by Patir and Cheng ${ }^{35}$ that concentrated on the macroscopic form of the Reynolds equation, which was later generalized to the anisotropic setting in the works of Tripp ${ }^{33}$ and Elrod. ${ }^{42}$ On the other hand, a similar anisotropic generalization for the tractions does not seem to be addressed until the work of Prat et al, ${ }^{34}$ which, however, omits the contributions to $\mathcal{T}^{ \pm}$from $\mathcal{T}_{p}^{ \pm}$. The homogenization analysis of Sahlin et $\mathrm{al}^{43}$ likewise addresses anisotropy but not $\mathcal{T}_{p}^{ \pm}$. Therefore, the homogenization-based analysis of Benhaboucha et $\mathrm{a}^{40}$ appears to be the first fully anisotropic formulation in the literature that also addresses $\mathcal{T}_{p}^{ \pm}$. Despite this result, homogenization-based traction formulations were employed only in a very limited number of studies, for instance, under different simplifying assumptions in the work of Buscaglia et $\mathrm{al}^{44}$ and Lukkassen et al. ${ }^{45}$

Although outside the scope of this study, it is remarked that many studies also incorporate the influence of microscopic contact, beginning with the original works by Patir and Cheng, ${ }^{35,41}$ and this aspect continues to be a numerical challenge. ${ }^{46-48}$ Among these studies, Persson ${ }^{49}$ presented an approach where rough contacts were resolved together with induced long range elastic deformations and the anisotropic macroscopic Reynolds equation was investigated under these conditions. The stress factors were then incorporated into this setting in the work of Persson and Scaraggi ${ }^{50}$ in a macroscopically isotropic setting, where $\mathcal{T}_{p}^{ \pm}$was also addressed, and a related anisotropic generalization was subsequently discussed in the work of Scaraggi et al. ${ }^{51}$ A more detailed discussion of the anisotropic traction formulation may be found in another work of Scaraggi et al, ${ }^{52}$ which recovers similar expressions to those which may be found in the work of Benhaboucha et al..$^{40}$ These studies, including the aforementioned work, ${ }^{40}$ are carried out in a simplified setting where only one surface is microscopically nonsmooth (see Section 4). Consequently, the homogenization-based traction expressions presented earlier are generalizations of these results to the case where both surfaces could be nonsmooth. Moreover, the dissipation expression forms the basis of a thermodynamic consistency argument (see Section 4), which, to our best knowledge, is discussed for the first time. Finally, it should also be noted that homogenization is naturally set in a periodic setting, whereas averaging is usually set in a random setting. For this reason, most of the averaging-based studies address random roughness and exact approaches such as the work of Persson and Scaraggi ${ }^{50}$ may not directly apply to 
the periodic setting, requiring approximate averaging approaches to be developed instead (see the work of Scaraggi ${ }^{53}$ for a recent discussion). This further highlights the importance of a general homogenization-based dissipation analysis for micro-textured interfaces.

\section{4 | UNILATERAL SETTING}

Homogenization eliminates rapid spatial fluctuations through space-averaging but rapid temporal fluctuations are still present and are represented by the dependence of $p_{0}$ on $\tau$, and similarly for all quantities that are related to $p_{0}$, which must be addressed within a macroscopic computation. This dependence may also be eliminated by time-averaging over the period $\mathcal{T}^{34,54}$ to extract a mean response with respect to absolute time $t_{\varepsilon}$ but it also disappears in a number of scenarios without the need for further manipulation ${ }^{32,54}$ : (1) when only one surface is microscopically nonsmooth, or (2) when the microscopic surface geometries are incommensurate. The latter is encountered when (a) the two surfaces have random roughness, or (b) they have a mismatch in their periodic textures, for instance if the ratio of the periodicities is an irrational number. Case (a) has implicitly enabled the consideration of random roughness on both surfaces, starting with the seminal studies in related works ${ }^{33,35,41,42}$ (see also the work of Prat et $\mathrm{al}^{34}$ ). This study focuses on periodic micro-texture design and optimization on a unit-cell of the interface. In order to avoid additional computation cost due to microscopic time dependence or due to incommensurate periodic micro-textures on both surfaces, only one surface will be endowed with a micro-texture (the more general cases are presently open problems). This micro-texture may be on either surface (top or bottom) and both surfaces can be moving. However, one can show that the results of an homogenization analysis applied to a simpler scenario where the nonsmooth surface is stationary and the smooth one moves can be reused to address the general scenario based on the foregoing results (for the computation of the fluid flux, see the work of Waseem et $\mathrm{al}^{1}$ and, for the tractions, see the work of Scaraggi et $\mathrm{al}^{52}$ ). In any case, the homogenized response will not display microscopic temporal fluctuations: $p_{0}=p_{0}(\boldsymbol{x}, t)$.

The simple scenario to be considered in this study (Figure 2) is where $\mathcal{P}^{+}$is microscopically smooth and geometrically coincident with $\mathcal{L}$, ie, $n_{0}^{+}=0=h^{+}$, although it moves with a tangential and/or normal velocity. On the other hand, $\mathcal{P}^{-}$has a both macroscopically and microscopically varying geometry, ie, $-h_{0}=n_{0}^{-} \neq 0 \neq h^{-}$, but is stationary in both tangential and normal directions. Defining $\boldsymbol{U}^{+}=\overline{\boldsymbol{U}}$ and $h^{-}=\bar{h}$ for simplicity, one obtains

$$
\boldsymbol{U}=\boldsymbol{V}=\overline{\boldsymbol{U}} \quad, \quad h_{\varepsilon}=h_{0}-\bar{h}
$$

For the microscopic sensitivity analysis to be carried out in the following section, it is necessary to state the forms of central results based on the simplifications noted earlier. For a complete picture, some of the unmodified expression will also be restated. As before, for brevity, some straightforward manipulations are not explicitly shown. To begin with, the cell problems read

$$
\left\langle(a \boldsymbol{I}+a \lambda) \nabla_{\boldsymbol{y}} \phi\right\rangle=0 \quad, \quad\left\langle(b \boldsymbol{I}+a \boldsymbol{\Lambda}) \nabla_{\boldsymbol{y}} \Phi\right\rangle=0
$$

FIGURE 2 The geometry of the micro-textured interface in the unilateral setting [Colour figure can be viewed at wileyonlinelibrary.com]

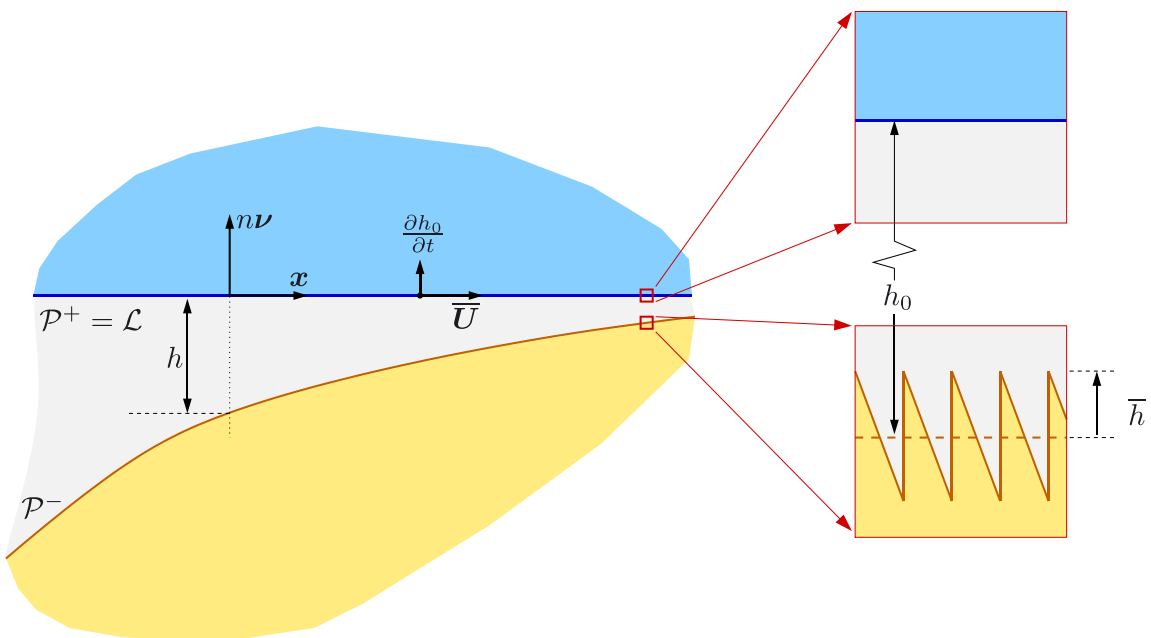


and the macroscopic flux $\boldsymbol{Q}(\boldsymbol{x}, t)$ is

$$
Q=-A G+C \bar{U}
$$

where the following flow factor tensors have been defined:

$$
\boldsymbol{A}=\left\langle a \boldsymbol{I}+a \lambda^{T}\right\rangle \quad, \quad \boldsymbol{C}=\left\langle b \boldsymbol{I}+a \boldsymbol{\Lambda}^{T}\right\rangle .
$$

The macroscopic interface dissipation only requires the knowledge of $\mathcal{T}^{+}$, which will be denoted by $\overline{\mathcal{T}}$. Also noting that $\frac{\partial h_{0}}{\partial t}=W^{+}$holds in this case, one obtains

$$
\Pi=\int_{\partial \mathcal{L}}\left(-p_{0}\right) \boldsymbol{Q} \cdot \boldsymbol{m} \mathrm{d} l+\int_{\mathcal{L}}^{\overline{\mathcal{T}}} \cdot \overline{\boldsymbol{U}} \mathrm{d} a+\int_{\mathcal{L}}\left(-p_{0}\right) \frac{\partial h_{0}}{\partial t} \mathrm{~d} a
$$

Upon making use of (25), one can also transform this expression into

$$
\Pi=\int_{\mathcal{L}}(-\boldsymbol{Q} \cdot \boldsymbol{G}+\overline{\mathcal{T}} \cdot \overline{\boldsymbol{U}}) \mathrm{d} a
$$

Note that either the second or the third term in (34) would vanish if the motion of $\mathcal{P}^{+}$is only in the normal or tangential direction. Moreover, the macroscopic problems to be considered will employ homogeneous boundary conditions $p_{0}=0$ on $\partial \mathcal{L}$ so that the first term will eventually also vanish. The alternative expression (35) is preferable because it remains unmodified under such special cases.

The macroscopic traction has the form

$$
\overline{\mathcal{T}}=\mathcal{T}^{+}=\mathcal{T}_{v}^{+}=\boldsymbol{D G}+\boldsymbol{F} \overline{\boldsymbol{U}}
$$

where the following stress factor tensors have been defined:

$$
\boldsymbol{D}=\left\langle b \boldsymbol{I}+b \lambda^{T}\right\rangle \quad, \quad \boldsymbol{F}=\left\langle e \boldsymbol{I}-b \boldsymbol{\Lambda}^{T}\right\rangle .
$$

For completeness, the individual parts for the traction vector of $\mathcal{P}^{-}$are

$$
\mathcal{T}_{p}^{-}=p_{0} \nabla_{\boldsymbol{x}} h_{0}-\left\langle h \lambda^{T}\right\rangle \boldsymbol{G}+\left\langle h \boldsymbol{\Lambda}^{T}\right\rangle \overline{\boldsymbol{U}} \quad, \quad \mathcal{T}_{v}^{-}=\left\langle b \boldsymbol{I}+b \lambda^{T}\right\rangle \boldsymbol{G}-\left\langle e \boldsymbol{I}+b \boldsymbol{\Lambda}^{T}\right\rangle \overline{\boldsymbol{U}}
$$

and the sum delivers a simpler expression for the total traction

$$
\mathcal{T}^{-}=\nabla_{\boldsymbol{x}}\left(p_{0} h_{0}\right)-\overline{\mathcal{T}}
$$

\section{5 | DISSIPATION INEQUALITY}

In this section, the thermodynamic consistency of the two-scale formulation will be explicitly analyzed and verified. To this end, first note that the macroscopic vectorial quantities that govern the interface dynamics are $\{\boldsymbol{Q}, \overline{\mathcal{T}}\}$, both of which are driven by $\{\boldsymbol{G}, \overline{\boldsymbol{U}}\}$. The expressions relating these quantities may be summarized together in the form

$$
\left\{\begin{array}{c}
-\boldsymbol{Q} \\
\overline{\mathcal{T}}
\end{array}\right\}=\left[\begin{array}{cc}
\boldsymbol{A} & -\boldsymbol{C} \\
\boldsymbol{D} & \boldsymbol{F}
\end{array}\right]\left\{\begin{array}{l}
\boldsymbol{G} \\
\boldsymbol{U}
\end{array}\right\}
$$

For a more compact reference, one may also write

$$
\boldsymbol{J}=\boldsymbol{L H}
$$

where

$$
\boldsymbol{J}=\left\{\begin{array}{c}
-\boldsymbol{Q} \\
\overline{\mathcal{T}}
\end{array}\right\} \quad, \quad \boldsymbol{L}=\left[\begin{array}{cc}
\boldsymbol{A} & -\boldsymbol{C} \\
\boldsymbol{D} & \boldsymbol{F}
\end{array}\right] \quad, \quad \boldsymbol{H}=\left\{\begin{array}{l}
\boldsymbol{G} \\
\overline{\boldsymbol{U}}
\end{array}\right\}
$$

Now, associating $\phi$ and $\Phi$ with the vector field $\omega$ in (31) and transposing the results, one obtains

$$
\left\langle\lambda\left(a \boldsymbol{I}+a \lambda^{T}\right)\right\rangle=\mathbf{0} \quad, \quad\left\langle\lambda\left(b \boldsymbol{I}+a \boldsymbol{\Lambda}^{T}\right)\right\rangle=\mathbf{0},
$$


which may be added to (33) $)_{1}$ and (33) $)_{2}$, respectively, to obtain the alternative expressions

$$
\boldsymbol{A}=\left\langle(\boldsymbol{I}+\lambda)\left(a \boldsymbol{I}+a \lambda^{T}\right)\right\rangle \quad, \quad \boldsymbol{C}=\left\langle(\boldsymbol{I}+\boldsymbol{\lambda})\left(b \boldsymbol{I}+a \Lambda^{T}\right)\right\rangle .
$$

Alternatively, associating $\phi$ and $\Phi$ with the vector field $\boldsymbol{\Omega}$ in (31) and transposing only the result associated with $\phi$, one obtains

$$
\left\langle\boldsymbol{\Lambda}\left(a \boldsymbol{I}+a \lambda^{T}\right)\right\rangle=\mathbf{0} \quad, \quad\left\langle(b \boldsymbol{I}+a \boldsymbol{\Lambda}) \boldsymbol{\Lambda}^{T}\right\rangle=\mathbf{0} .
$$

Adding these to $(37)_{1}$ and (37) 2 , respectively, one obtains the alternative expressions

$$
\boldsymbol{D}=\left\langle(b \boldsymbol{I}+a \boldsymbol{\Lambda})(\boldsymbol{I}+\lambda)^{T}\right\rangle \quad, \quad \boldsymbol{F}=\left\langle e \boldsymbol{I}+a \mathbf{\Lambda} \mathbf{\Lambda}^{T}\right\rangle .
$$

Now, based on (44) and (46), it is clear that $\{\boldsymbol{A}, \boldsymbol{F}\}$ are symmetric positive definite tensors and it is also observed that $\boldsymbol{D}=\boldsymbol{C}^{T}$, although $\boldsymbol{C}$ is evidently not symmetric in general. ${ }^{1}$ Indeed, these observations are anticipated from the physics of the problem because, making use of (41), (35) now takes the form

$$
\Pi=\int_{\mathcal{L}} \boldsymbol{H} \cdot \boldsymbol{J} \mathrm{d} a=\int_{\mathcal{L}} \boldsymbol{H} \cdot \boldsymbol{L} \boldsymbol{H} \mathrm{d} a=\int_{\mathcal{L}}(\boldsymbol{G} \cdot \boldsymbol{A} \boldsymbol{G}+\overline{\boldsymbol{U}} \cdot \boldsymbol{F} \overline{\boldsymbol{U}}) \mathrm{d} a \geq 0 .
$$

Hence, the macroscopic dissipation associated with the hydrodynamically lubricated micro-textured interface is guaranteed to be (pointwise) nonnegative, thereby confirming the thermodynamic consistency of the two-scale formulation.

\section{SENSITIVITY ANALYSIS}

\section{1 | Micro-texture design and optimization}

The adaptation of topology optimization to micro-texture design in a homogenization-based setting was first realized in the work of Waseem et $\mathrm{al}^{1}$ with an emphasis on microscopic objective functions and, subsequently, employed via two-scale analysis in another work of Waseem et al, ${ }^{2}$ where the micro-texture evolution was driven by macroscopic objective functions. Presently, the aim is to follow these studies by examining the influence of dissipation on optimal micro-textures. For brevity, only a restricted class of topology descriptions will be adapted (for a range of possibilities, see the work of Waseem et $\mathrm{al}^{1}$ ) Specifically, the micro-texture will be discretized by a finite element mesh with $N_{m}$ elements per direction to solve the cell problems (31). An element-wise constant design variable distribution $s$ is assigned to this mesh, with degrees of freedom $s^{I} \in[0,1]\left(I=1\right.$ to $\left.N_{m}^{2}\right)$. However, if such a distribution is directly employed in the description of the micro-texture geometry, the designs are prone to checkerboard patterns. ${ }^{55,56}$ This is typically avoided by employing filters (see the works of Sigmund ${ }^{57}$ and Svanberg and Svärd ${ }^{58}$ for reviews). Presently, the design variable degrees of freedom will be filtered using a linear filter ${ }^{59,60}$ to obtain a morphology variable distribution $\rho$, with element-wise constant degrees of freedom $\rho^{I} \in[0,1]$. At a given macroscopic point with film thickness $h_{0}(\boldsymbol{x}, t)$, the micro-textured surface $\bar{h} \in\left[\bar{h}_{\min }, \bar{h}_{\max }\right]$ is now described as the distribution, ${ }^{61,62}$ ie,

$$
\bar{h}=\bar{h}_{\min }+\left(\bar{h}_{\max }-\bar{h}_{\min }\right) \rho,
$$

thereby delivering $h=h_{0}-\bar{h}$ for the calculation of the coefficients $\{a, b, e\}$, which appear in the homogenization analysis. With these choices for the filter and the particular description of $\bar{h}$, the topology optimization framework is suitable for obtaining both smoothly varying surface geometries as well as comparatively sharper ones. The sharpness of the transitions in $\bar{h}$ across the unit-cell is governed by the objective function formulation, as well as by the radius $R$ of the linear filter. Sharper transitions, hence micro-textures that can deliver more extreme macroscopic responses, may be achieved by replacing $\rho$ with $\rho^{3}$ in (48) and employing a suitable filter. ${ }^{1}$ Note that this latter choice is more natural in material design, where individual design components are separated by sharp interfaces, but there is no such necessity in surface design where smoothness may actually be desirable.

As a consequence of the micro-texture design space, any microscopic or macroscopic objective $\varphi$ is indirectly a function of the $s$-distribution. Note that this distribution is indirectly constrained due to the restriction $\chi(s)=\langle\bar{h}\rangle=0$. All optimization problems will be formulated so as to minimize $\varphi(s)$ subject to the equality constraint $\chi(s)=0$ while ensuring $s \in[0,1]$. If the problem requires the maximization of a function then its negative is employed as the objective in 
this minimization setting. The minimization is carried out using the method of moving asymptotes, ${ }^{63}$ which requires the sensitivity of the objective and the equality constraint. These are discussed next.

\section{2 | Microscale}

The film thickness $h$ in general depends on $\{\boldsymbol{x}, \boldsymbol{y}, t\}$. In view of (48), the variation of $h$ with $\boldsymbol{y}$ is now controlled by the design variable degrees of freedom $s^{I}$, which (indirectly) describe the micro-texture geometry. Using the sensitivity notation $(\cdot)^{\prime}=\frac{\partial(\cdot)}{\partial s^{I}}$ for a particular choice of $s^{I}$, one may show that the sensitivities of $\boldsymbol{A}$ and $\boldsymbol{C}=\boldsymbol{D}^{T}$ take the forms ${ }^{1}$

$$
\boldsymbol{A}^{\prime}=\left\langle(\boldsymbol{I}+\lambda)\left(a^{\prime} \boldsymbol{I}+a^{\prime} \boldsymbol{\lambda}^{T}\right)\right\rangle \quad, \quad \boldsymbol{C}^{\prime}=\left\langle(\boldsymbol{I}+\boldsymbol{\lambda})\left(b^{\prime} \boldsymbol{I}+a^{\prime} \Lambda^{T}\right)\right\rangle
$$

These microscopic sensitivity results are numerically advantageous because not only it is not necessary to explicitly compute $\lambda^{\prime}$ or $\Lambda^{\prime}$ but also the forms of the sensitivity expressions follow the original forms in (44). It is now shown that the sensitivity of $\boldsymbol{F}$ also takes a similarly advantageous form. For this purpose, an alternative $\boldsymbol{F}$ expression is first formulated by subtracting the transpose of $(45)_{2}$ from $(37)_{2}$, where positive definiteness is not immediately observed, yet symmetry is evident:

$$
\boldsymbol{F}=\left\langle e \boldsymbol{I}-b\left(\boldsymbol{\Lambda}+\boldsymbol{\Lambda}^{T}\right)-a \boldsymbol{\Lambda} \boldsymbol{\Lambda}^{T}\right\rangle .
$$

The sensitivity result to be shown is

$$
\boldsymbol{F}^{\prime}=\left\langle e^{\prime} \boldsymbol{I}-b^{\prime}\left(\boldsymbol{\Lambda}+\boldsymbol{\Lambda}^{T}\right)-a^{\prime} \mathbf{\Lambda} \mathbf{\Lambda}^{T}\right\rangle .
$$

Now, the direct sensitivity of $(46)_{2}$ is

$$
\boldsymbol{F}^{\prime}=\left\langle e^{\prime} \boldsymbol{I}+a^{\prime} \boldsymbol{\Lambda} \boldsymbol{\Lambda}^{T}+a \boldsymbol{\Lambda}^{\prime} \boldsymbol{\Lambda}^{T}+a\left(\boldsymbol{\Lambda}^{\prime} \boldsymbol{\Lambda}^{T}\right)^{T}\right\rangle .
$$

On the other hand, the sensitivity of $(31)_{2}$ is

$$
\left\langle\left(b^{\prime} \boldsymbol{I}+a^{\prime} \boldsymbol{\Lambda}+a \boldsymbol{\Lambda}^{\prime}\right) \nabla_{\boldsymbol{y}} \Phi\right\rangle=0,
$$

where the fact that $\Phi^{\prime}$ also qualifies as a test function has been employed. Now, associating $\Phi$ with the vector field $\mathbf{\Omega}$, this result delivers

$$
\left\langle a \boldsymbol{\Lambda}^{\prime} \boldsymbol{\Lambda}^{T}\right\rangle=-\left\langle b^{\prime} \boldsymbol{\Lambda}^{T}+a^{\prime} \boldsymbol{\Lambda} \mathbf{\Lambda}^{T}\right\rangle,
$$

which, when employed in (52), delivers (51) as desired. The quantities $\left\{a^{\prime}, b^{\prime}, e^{\prime}\right\}$ are easily evaluated, once the particular form of $h=h(s)$ is specified (Section 6.1).

\section{3 | Macroscale}

In order to address central numerical issues in macroscopic sensitivity analysis, two representative macroscopic quantities are considered in this section. The analysis of closely related objective functions that will be specified during the numerical investigations may be carried out in a similar manner. Because $h$ was parametrized by a variable $s^{I}$ in Section 6.2, a macroscopic quantity such as the load capacity $L_{0}$ of the interface now also depends on $s^{I}$ as well, ie,

$$
L_{0}=\int_{\mathcal{L}} p_{0} \mathrm{~d} a \Longrightarrow L_{0}^{\prime}=\int_{\mathcal{L}} p_{0}^{\prime} \mathrm{d} a .
$$

Here, $p_{0}^{\prime}$ may be computed through the sensitivity of (25), by making use of (32):

$$
-\nabla_{\boldsymbol{x}} \cdot\left(-\boldsymbol{A}^{\prime} \boldsymbol{G}-\boldsymbol{A} \boldsymbol{G}^{\prime}+\boldsymbol{C}^{\prime} \overline{\boldsymbol{U}}\right)=0
$$

In view of the results in Section 6.2, the only unknown to be solved for here is the $p_{0}^{\prime}$ in $G^{\prime}=\nabla_{x} p_{0}^{\prime}$, after which $L_{0}^{\prime}$ may be evaluated. Because $L_{0}^{\prime}=\frac{\partial L_{0}}{\partial s^{I}}$, this solution must be repeated for each $s^{I}$. Micro-texture optimization via two-scale analysis based on this direct sensitivity approach was carried out recently in the work of Waseem et al. ${ }^{2}$ As discussed therein, the cost of evaluating $L_{0}^{\prime}$ may be reduced by employing an adjoint method but, in view of the dominating cost of microscopic 
analysis in the two-scale optimization problems to be considered, the direct approach will be preferred for macroscopic sensitivity analysis due to its straightforward application to different macroscopic objectives. Therefore, the macroscopic dissipation is also analyzed in a similar manner. Employing the form in (47), one obtains

$$
\Pi^{\prime}=\int_{\mathcal{L}}\left(2 \boldsymbol{G}^{\prime} \cdot \boldsymbol{A} \boldsymbol{G}+\boldsymbol{G} \cdot \boldsymbol{A}^{\prime} \boldsymbol{G}+\overline{\boldsymbol{U}} \cdot \boldsymbol{F}^{\prime} \overline{\boldsymbol{U}}\right) \mathrm{d} a,
$$

which may be evaluated based on the results obtained so far without the need for further analysis. Note that the design space will eventually be the same for both microscopic and macroscopic objectives because optimization will always be carried out over a single unit-cell, which describes the periodic micro-texture. The main challenge associated with macroscopic objectives is the computational cost of the two-scale analysis.

\section{7 | NUMERICAL INVESTIGATIONS}

\section{1 | Problem settings and major parameters}

The problem settings and the values of various simulation parameters will be borrowed from the works of Waseem et al. ${ }^{1,2}$ The viscosity is always set to $\mu=0.14 \mathrm{~Pa} \cdot \mathrm{s}$. On the microscale, the square unit-cell $\mathcal{Y}$ will be discretized using $N_{m}=40$ bilinear elements per edge. The radius of the linear filter is $R=4$. The measurement of distance in $\mathcal{Y}$ and therefore the reported value of the filter radius are both normalized by the edge length of an element because the absolute size of $\mathcal{Y}$, characterized by its edge length $\ell_{m}$, does not influence the homogenized response in the present setting. The values of $\left\{\bar{h}_{\text {min }}, \bar{h}_{\text {max }}\right\}$ will be specified later. All lengths are in $\mu \mathrm{m}$, velocities are in $\mu \mathrm{m} / \mathrm{s}$, and $\boldsymbol{G}$ is in $\mathrm{Pa} / \mu \mathrm{m}$ (their units will not be explicitly indicated further). Note that all figures that depict the surface topology employ a color scheme that corresponds to the $\bar{h}$-distribution, with red indicating peaks $\left(\bar{h}_{\max }\right)$ and blue indicating valleys $\left(\bar{h}_{\text {min }}\right)$. Starting with Figure 3 , the color legend is not explicitly shown in such plots for brevity. Three-dimensional figures of the surface topology will explicitly indicate the values of $\left\{\bar{h}_{\min }, \bar{h}_{\max }\right\}$. Both two- and three-dimensional depictions of the $\bar{h}$-distribution are included in Figure 4 to clarify the color scheme as well as to help visualize the link between these two types of depictions for typical micro-textures designs that will be obtained in the example studies. Note that, although optimization employs a single unit-cell, a $3 \times 3$ tiling of the unit-cell is preferred in the graphical depiction of the optimization results for a more clear visual representation of the micro-texture geometry.

On the macroscale, the square interface domain $\mathcal{L}$ is assigned an edge length $\ell_{M}=200$ and will be discretized using $N_{M}=20$ bilinear elements per edge to obtain the macroscopic solution $p_{0}$. The Gauss-Legendre integration on this mesh is carried out with $N_{G} \times N_{G}$ points, where $N_{G}=2$. When $h_{0}$ varies with time, a total number of $N_{T}=10$ time steps will be employed to discretize this variation. Hence, in general, a separate microscopic analysis must be carried out at each of the $\left(N_{M} N_{G}\right)^{2}$ points of the interface at each of the $N_{T}+1$ points in time to address, respectively, the spatial and temporal variations in flow and stress factor tensors. Although strategies may be devised to alleviate the significant two-scale optimization cost associated with a spatial variation, ${ }^{2,64}$ only temporal variations will be addressed within the macroscopic problem setup of this work. Specifically, the classical squeeze-film problem ${ }^{24}$ will first be considered where $\mathcal{P}^{-}$is parallel to $\mathcal{P}^{+}$and $\mathcal{P}^{+}$moves only in the normal direction such that $h_{0}$ varies from $h_{0}^{\max }=2.5$ down to $h_{0}^{\min }=1$ and then back to its original value to complete a cycle, with a velocity profile $\frac{\partial h_{0}}{\partial t}=-\sin (2 \pi t / T)$ and a period $T=1.5 \pi$. In view of $(25)$ and (32), subject to homogeneous boundary conditions $p_{0}=0$ on $\partial \mathcal{L}$, this setup requires the computation of only $\boldsymbol{A}$. Therefore, in order to also reflect the influence of $\boldsymbol{F}$, a modified squeeze-film problem will subsequently also be considered such that a tangential velocity $\bar{U}$ is additionally assigned to $\mathcal{P}^{+}$. This motion does not influence the macroscopic pressure distribution for a given micro-texture because $\boldsymbol{F}$ and $\overline{\boldsymbol{U}}$ are constants across the interface but it will affect the micro-texture design due to its contribution to the total dissipation (47).

Based on these numerical choices and following the terminology in Section 1, the next sections present a series of MICOO and MACOO investigations.

\section{2 | Microscopic objective optimization}

\subsection{1 | Individual dissipation contributions}

For MiCOO, the main objective function of interest is the pointwise dissipation from (47), ie,

$$
\varphi=\underbrace{\boldsymbol{G} \cdot \boldsymbol{A} \boldsymbol{G}}_{\varphi^{A}}+\underbrace{\overline{\boldsymbol{U}} \cdot \boldsymbol{F} \overline{\boldsymbol{U}}}_{\varphi^{F}} .
$$


In order to assess the behavior of the individual contributions $\left\{\varphi^{A}, \varphi^{F}\right\}$ in this objective function, as well as to be able to interpret the changes in the micro-texture designs that will be obtained in upcoming examples, they are first considered separately in a series of optimization studies, which are summarized in Figure 3. Here, either the maximization or the minimization of these contributions is carried out while the angle of $\boldsymbol{G}$ for $\varphi^{A}$ and $\overline{\boldsymbol{U}}$ for $\varphi^{F}$ is varied. It is observed that the minimization of $\varphi^{A}$ delivers qualitatively similar micro-textures to the maximization of $\varphi^{F}$. This similarity arises despite the difference in optimization direction due to the fact that $\boldsymbol{A}$ only contains $h^{3}$-related terms (see (33) $)_{1}$, whereas $\boldsymbol{F}$ also contains an $h^{-1}$-related term (see $(37)_{2}$ ). Furthermore, as one might expect, the maximization of $\varphi^{A}$ leads to micro-textures that are qualitatively the opposite of those that are obtained from the minimization of $\varphi^{A}$, in particular with respect to
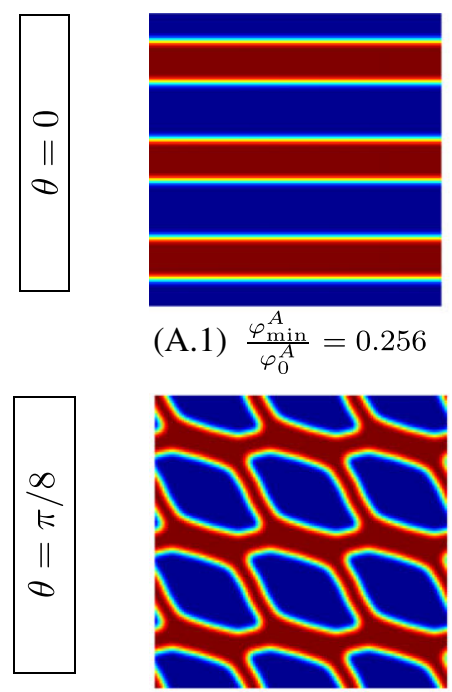

(A.2) $\frac{\varphi_{\min }^{A}}{\varphi_{0}^{A}}=0.326$
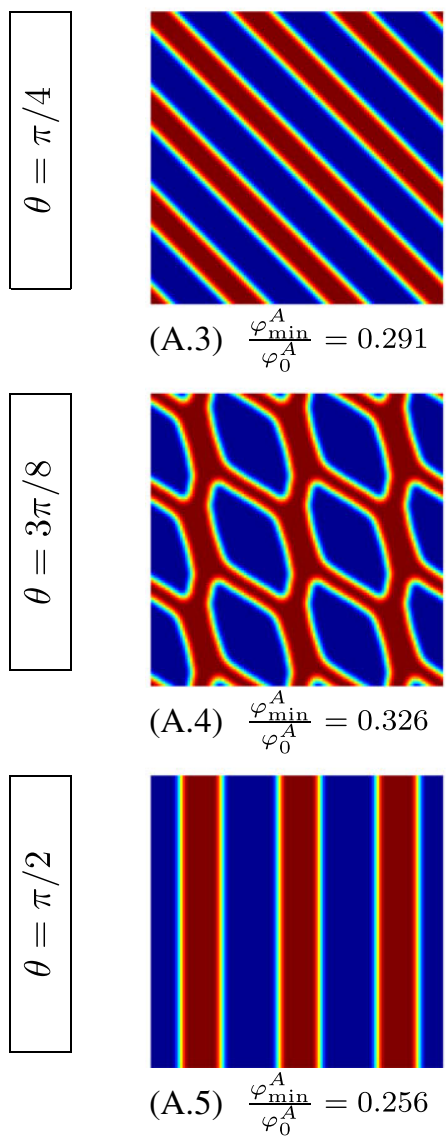
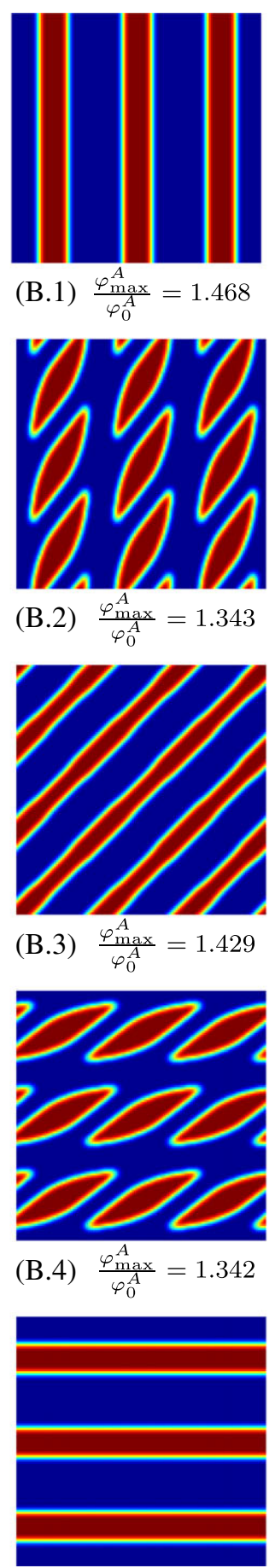

(B.5) $\frac{\varphi_{\max }^{A}}{\varphi_{0}^{A}}=1.468$
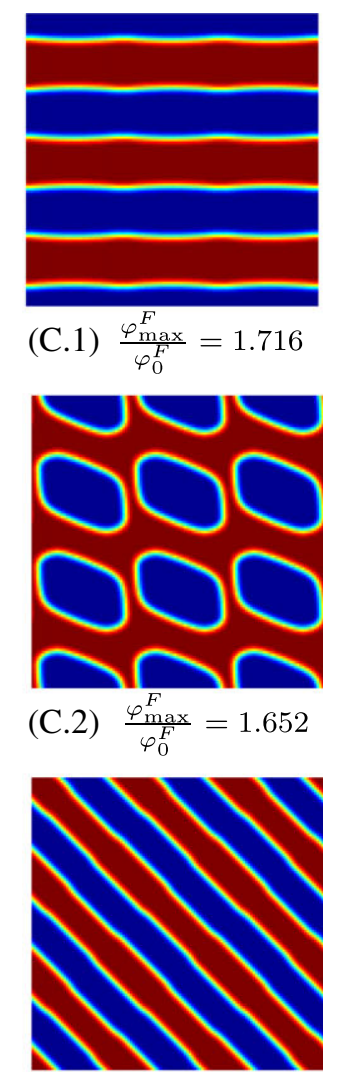

(C.3) $\frac{\varphi_{\max }^{F}}{\varphi_{0}^{F}}=1.686$
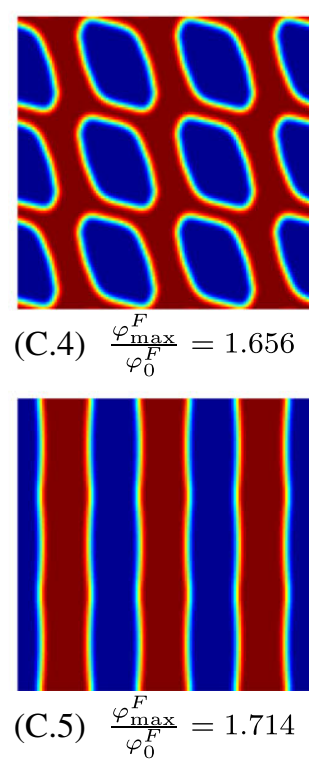

FIGURE 3 The objective functions $\varphi^{A}$ and $\varphi^{F}$ from (58) are employed in microscopic objective optimization (MICOO) studies: (A) minimization of $\varphi^{A}$, (B) maximization of $\varphi^{A}$, and (C) maximization of $\varphi^{F}$. The angle $\theta$ (clockwise from the vertical axis) indicates the direction of $\boldsymbol{G}$ or $\overline{\boldsymbol{U}}$ (both with unit magnitude).

$\left\{h_{0}, \bar{h}_{\min }, \bar{h}_{\max }\right\}=\{2.2,-0.8,1.2\}$ was used in $(\mathrm{A}, \mathrm{B})$ and $\left\{h_{0}, \bar{h}_{\min }, \bar{h}_{\max }\right\}=\{2,-1,1\}$ in $(\mathrm{C})$ 
the nature of the surface topology as well as its direction. For instance, for an angle of $\theta=3 \pi / 8$, minimization delivers holes on a surface, whereas maximization delivers bumps, and the direction of these features visually differ by $\pi / 2$. In all cases, the minimization or maximization values for $\varphi^{A}$ and $\varphi^{F}$ differ significantly from the homogeneous surface values $\varphi_{0}^{A}=a_{0}\|\boldsymbol{G}\|^{2}$ and $\varphi_{0}^{F}=e_{0}\|\overline{\boldsymbol{U}}\|^{2}$, which highlight the strong influence of surface texturing.

It is noted that micro-texture designs associated with the minimization of $\varphi^{F}$ are not displayed because the minimizing texture is always a homogeneous surface $\left(h=h_{0}\right)$. In fact, this may be shown by proving that $\varphi^{F} \geq \varphi_{0}^{F}$. For this purpose, using $(46)_{2}$, one may write

$$
\varphi^{F}=\overline{\boldsymbol{U}} \cdot \boldsymbol{F} \overline{\boldsymbol{U}}=\langle e\rangle\|\overline{\boldsymbol{U}}\|^{2}+\left\langle a\left(\Lambda^{T} \overline{\boldsymbol{U}}\right) \cdot\left(\Lambda^{T} \overline{\boldsymbol{U}}\right)\right\rangle .
$$

Both contributions are nonnegative and the second one vanishes only for $\boldsymbol{\Lambda}=\mathbf{0}$, which is only possible for $h=h_{0}$ in the cell problems of Section 3. To complete the proof, it is therefore sufficient to show that $\langle e\rangle \geq e_{0}$. Recalling the definition (7) ${ }_{2}$ of $e$ as well as $h_{0}=\langle h\rangle$, this requirement is equivalent to $\langle h\rangle^{-1} \geq\langle h\rangle^{-1}$, or $\langle h\rangle \geq\left\langle h^{-1}\right\rangle^{-1}$. This latter inequality indeed follows from the fact that the arithmetic average of $h>0$ is never less than its harmonic average. Hence, the textured surface can never have a $\varphi^{F}$ value that is less than the one for a homogeneous surface.

\subsection{2 | Total dissipation}

Based on the preceding discussion, it may be stated that the maximization of $\varphi=\varphi^{A}+\varphi^{F}$ is a competition among two terms, which individually deliver qualitatively opposing micro-textures with respect to surface topology and orientation. On the other hand, the minimization problem is a competition among two terms, one of which always delivers a homogeneous surface as the optimum. In order to demonstrate how this competition influences the micro-texture design when $\varphi$ is the objective function, a MICOO study is carried out where only the magnitude of $\boldsymbol{G}$ is varied (Figure 4). In the case of minimization, when $\|\boldsymbol{G}\|$ is sufficiently high, $\varphi^{A}$ dominates $\varphi$ so that the optimization result is qualitatively closer to the micro-texture that one would obtain from the direct minimization of $\varphi^{A}$, compare, for instance, Figure 4(a-1) with Figure 3(a-2). On the other hand, with decreasing $\|\boldsymbol{G}\|$, the optimization result approaches toward a homogeneous surface because the $\varphi^{F}$ starts to dominate the value of $\varphi$ and the direct minimization of $\varphi^{F}$ always delivers a homogeneous surface. In the maximization study, at large values of $\|\boldsymbol{G}\|, \varphi^{A}$ and $\varphi^{F}$ are of comparable magnitude, although the optimization outcome is qualitatively closer to that which would be obtained with $\varphi^{A}$ alone, compare, for instance, Figure 4(b-1) with Figure 3(b-2). With decreasing $\|\boldsymbol{G}\|$, similar to the minimization problem, $\varphi^{F}$ starts to dominate the value of $\varphi$ such that the micro-texture design becomes qualitatively similar to that which would be obtained with $\varphi^{F}$ alone, compare, for instance, Figure 4(b-5) with Figure 3(c-2). Note that, in Figure 4(b-1), the holes on the surface are connected, whereas, in Figure 4(b-5), the bumps are connected. Therefore, Figure 4(b-3) represents a transition topology where both are connected.

\subsubsection{Interface parameters}

In order to assess the influence of various interface parameters on the micro-texture design, a series of MicOO studies will be carried in this section. As demonstrated earlier in Figure 4, maximization of $\varphi=\varphi^{A}+\varphi^{F}$ leads to a very clear transition between two types of textures, depending on whether $\varphi^{A}$ or $\varphi^{F}$ dominates. For this reason, the objective will be the maximization of $\varphi$ in the following examples as well.

Figure 5 summarizes the influence of changing $h_{0}$ together with $\left\{\bar{h}_{\min }, \bar{h}_{\max }\right\}$ in such a way that the maximum $\left(h_{\max }\right)$ and minimum $\left(h_{\min }\right)$ values of $h=h_{0}-\bar{h}$ remains fixed. The starting value of $h_{0}$ as well as $\{\boldsymbol{G}, \overline{\boldsymbol{U}}\}$ are chosen to deliver balanced values of $\varphi^{A}$ and $\varphi^{F}$. Such a parametric change is then observed to only lead to a variation in the area fraction of the features that are observed in the micro-texture design because $\langle h\rangle=h_{0}$ changes while $\left\{h_{\min }, h_{\max }\right\}$ remain fixed. The surface topology does not display stronger changes because the $\varphi^{A}$ and $\varphi^{F}$ contributions remain relatively well balanced. On the other hand, when $h_{0}$ alone is changed while $\left\{\bar{h}_{\min }, \bar{h}_{\text {max }}\right\}$ are held fixed, this corresponds to varying the distance of the textured surface away from the flat and stationary one. As noted before, $\boldsymbol{A}$ contains only $h^{3}$-related terms, whereas $\boldsymbol{F}$ also contains an $h^{-1}$-related term. Therefore, $\varphi^{F}$ starts to dominate $\varphi$ when $h_{0}$ decreases and a transition in the micro-texture design is observed, as summarized in Figure 6, similar to the maximization study in Figure 4.

Another similar transition may be observed if the direction of only one vector is varied while all other parameters are held fixed. In Figure 7, the angle associated with $\overline{\boldsymbol{U}}$ is gradually increased. At all values, $\varphi^{A}$ and $\varphi^{F}$ remain well-balanced so that a strong transition in surface topology is not observed. Nevertheless, the orientation of the design features shift toward a vertical alignment, which, based on the observations of Figure 3, is the one that would be preferred when $\varphi^{F}$ significantly dominates $\varphi$. 

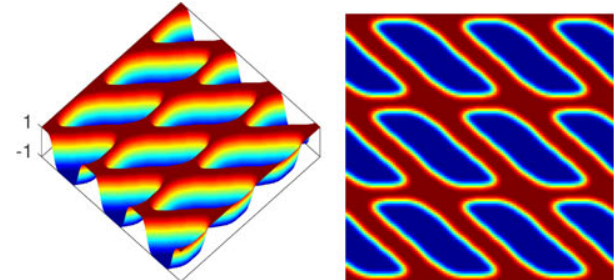

(A.1)
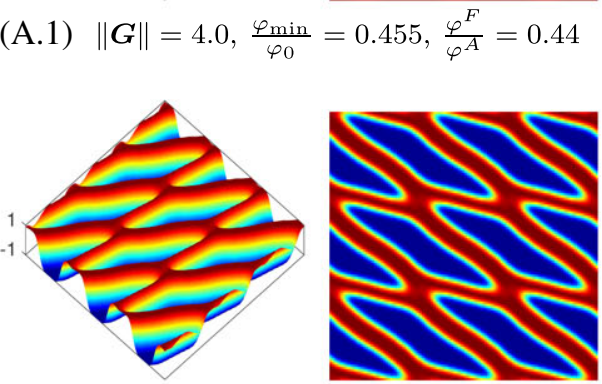

(A.2)
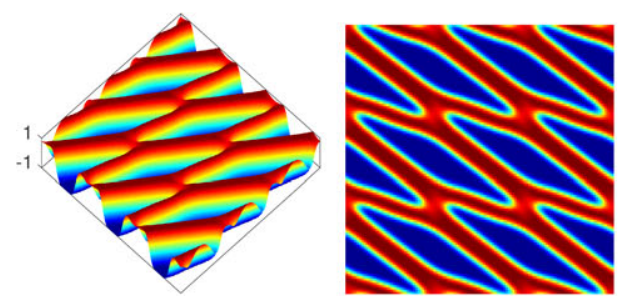

(A.3) $\|\boldsymbol{G}\|=1.8, \frac{\varphi_{\min }}{\varphi_{0}}=0.755, \frac{\varphi^{F}}{\varphi^{A}}=1.99$
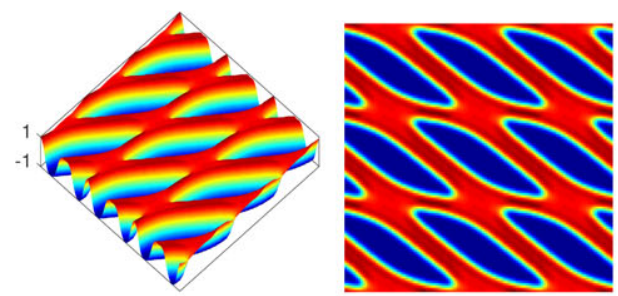

(A.4) $\|\boldsymbol{G}\|=1.5, \frac{\varphi_{\min }}{\varphi_{0}}=0.854, \frac{\varphi^{F}}{\varphi^{A}}=2.21$
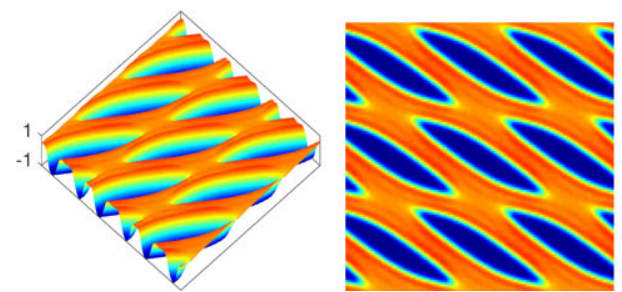

(A.5) $\|\boldsymbol{G}\|=1.2, \frac{\varphi_{\min }}{\varphi_{0}}=0.947, \frac{\varphi^{F}}{\varphi^{A}}=2.02$
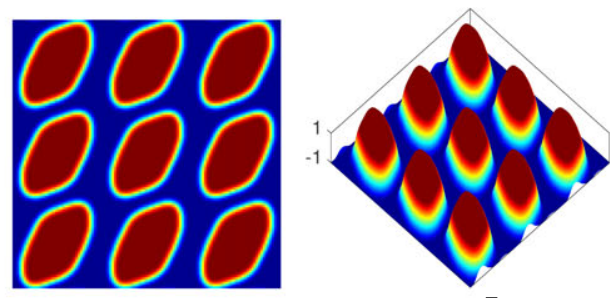

(B.1) $\|\boldsymbol{G}\|=0.8, \frac{\varphi_{\max }}{\varphi_{0}}=1.331, \frac{\varphi^{F}}{\varphi^{A}}=2.34$
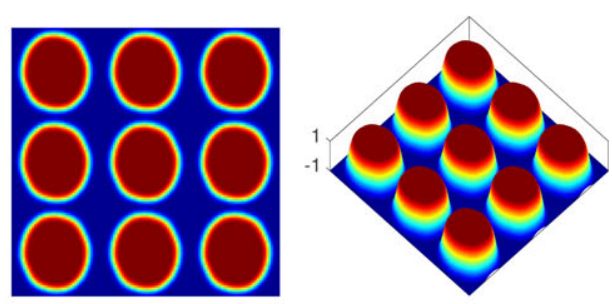

(B.2)
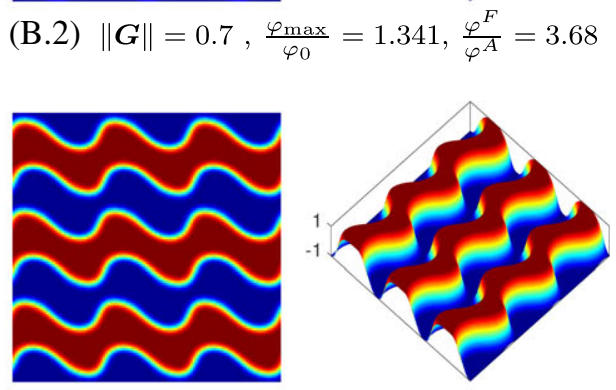

(B.3) $\|\boldsymbol{G}\|=0.6, \frac{\varphi_{\max }}{\varphi_{0}}=1.390, \frac{\varphi^{F}}{\varphi^{A}}=11.06$
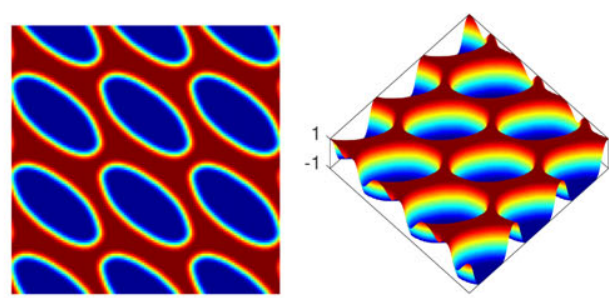

(B.4) $\|\boldsymbol{G}\|=0.5, \frac{\varphi_{\max }}{\varphi_{0}}=1.464, \frac{\varphi^{F}}{\varphi^{A}}=25.98$
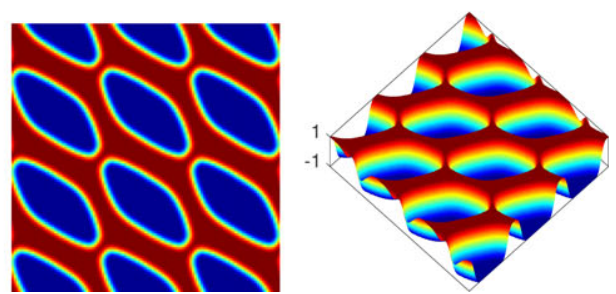

(B.5) $\|\boldsymbol{G}\|=0.4, \frac{\varphi_{\max }}{\varphi_{0}}=1.526, \frac{\varphi^{F}}{\varphi^{A}}=40.66$
FIGURE 4 The objective function $\varphi$ in (58) is (A) minimized or (B) maximized, with $\theta=\pi / 6$ as the direction of both $\overline{\boldsymbol{U}}$ and $\boldsymbol{G} .\|\overline{\boldsymbol{U}}\|=10$ is fixed and only the value of $\|\boldsymbol{G}\|$ is varied to assess the variation in the relative magnitudes of $\varphi^{A}$ and $\varphi^{F}$. In all cases,

$\left\{h_{0}, \bar{h}_{\min }, \bar{h}_{\max }\right\}=\{2,-1,1\}$

As noted in Section 6.1, the description of the morphology variable distribution also allows capturing smoothly varying surface geometries in addition to sharper ones. This is demonstrated in Figure 8, where, for a given set of interface parameters, $\varphi$ is driven to a particular value $\varphi^{*}$, which lies between the maximum and minimum values possible, by minimizing a new objective function $\frac{1}{2}\left(\varphi-\varphi^{*}\right)^{2}$. Near the homogeneous value $\varphi_{0}$, the surface topology becomes significantly smoother and less pronounced, whereas sharper features are obtained for the extremal designs.

Together with the examples that were presented in the previous sections, the examples of this section clearly demonstrate that the optimal surface topology is not necessarily described by simple geometrical features that may enable the use of approximate analytical approaches for both design and homogenization. Consequently, it is advantageous to work in a flexible design space that is offered by homogenization-based topology optimization, which can capture the optimal micro-texture design. 


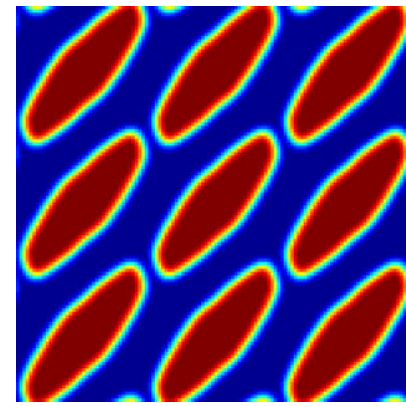

(A) $\delta=0.1, \frac{\varphi^{F}}{\varphi^{A}}=1.13$

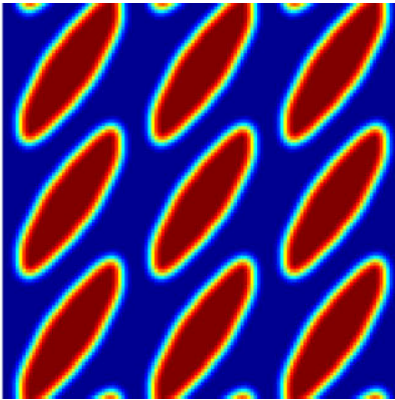

(B) $\delta=0.2, \frac{\varphi^{F}}{\varphi^{A}}=0.96$

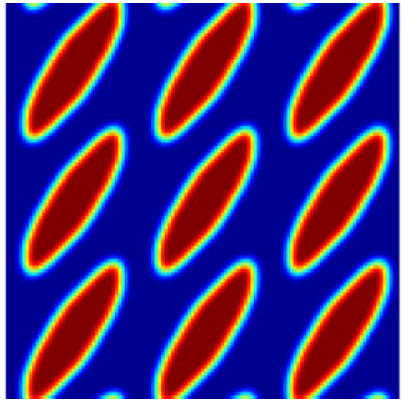

(C) $\delta=0.3, \frac{\varphi^{F}}{\varphi^{A}}=0.82$

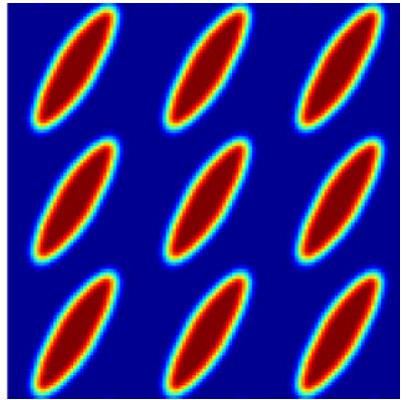

(D) $\delta=0.5, \frac{\varphi^{F}}{\varphi^{A}}=0.61$

FIGURE 5 For a fixed angle $(\theta=\pi / 6)$ for $\boldsymbol{G}$ and $\overline{\boldsymbol{U}}$ with magnitudes $\|\boldsymbol{G}\|=1$ and $\|\overline{\boldsymbol{U}}\|=10$, maximization of $\varphi=\varphi^{A}+\varphi^{F}$ is carried out while the value of $h_{0}$ is varied such that $h_{0}=2+\delta$ and $\left\{\bar{h}_{\min }, \bar{h}_{\max }\right\}=\{-1+\delta, 1+\delta\}$, which leads to fixed values of $\left\{h_{\min }, h_{\max }\right\}=\{1,3\}$

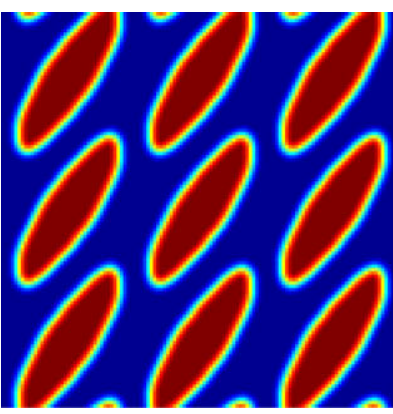

(A) $\delta=0, \frac{\varphi^{F}}{\varphi^{A}}=0.96$

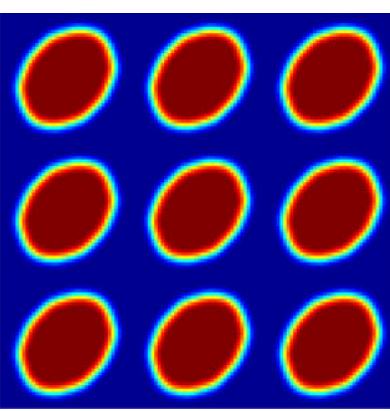

(B) $\delta=0.3, \frac{\varphi^{F}}{\varphi^{A}}=2.33$

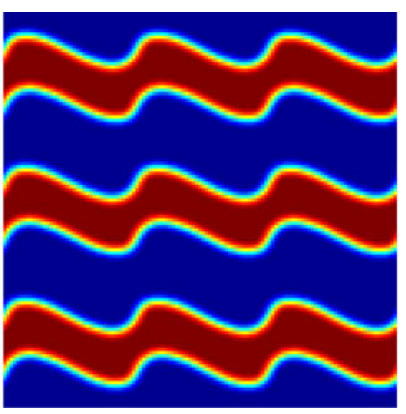

(C) $\delta=0.6, \frac{\varphi^{F}}{\varphi^{A}}=19.9$

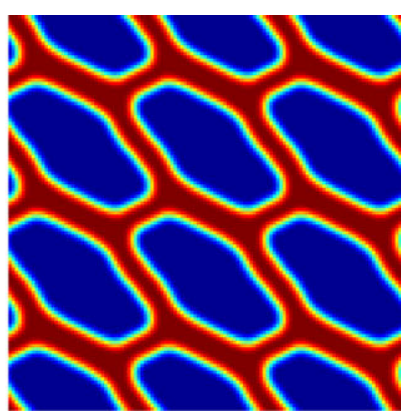

(D) $\delta=0.8, \frac{\varphi^{F}}{\varphi^{A}}=994$

FIGURE 6 For a fixed angle $(\pi / 6)$ for $\boldsymbol{G}$ and $\overline{\boldsymbol{U}}$ with magnitudes $\|\boldsymbol{G}\|=1$ and $\|\overline{\boldsymbol{U}}\|=10$, maximization of $\varphi=\varphi^{A}+\varphi^{F}$ is carried out while the value of $h_{0}$ is varied such that $h_{0}=2-\delta$ and $\left\{\bar{h}_{\min }, \bar{h}_{\max }\right\}=\{-1,1\}$ are fixed

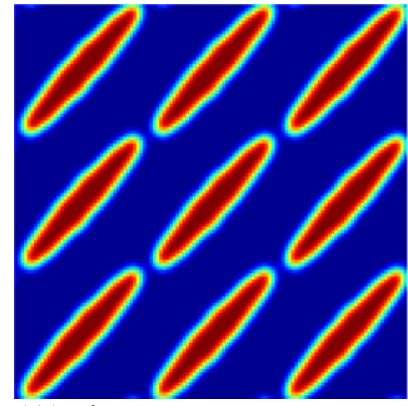

(A) $\theta=0$

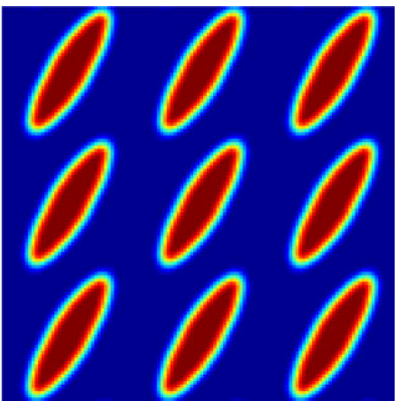

(B) $\theta=\pi / 6$

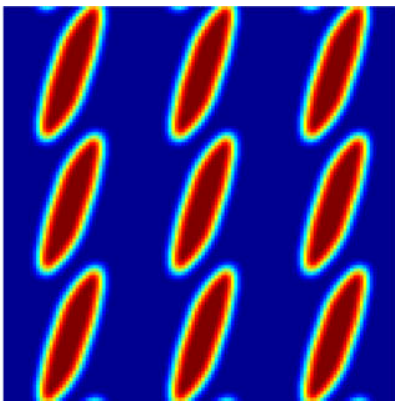

(C) $\theta=\pi / 3$

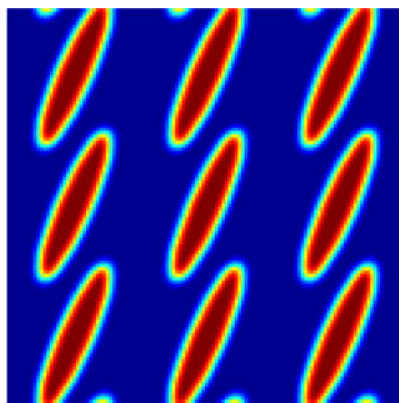

(D) $\theta=\pi / 2$

FIGURE 7 For a fixed angle $(\pi / 6)$ for $\boldsymbol{G}$ with magnitude $\|\boldsymbol{G}\|=1$, maximization of $\varphi$ is carried out while the value angle $\theta$ for $\overline{\boldsymbol{U}}$ with $\|\overline{\boldsymbol{U}}\|=10$ is varied. Here, $\left\{h_{0}, \bar{h}_{\min }, \bar{h}_{\max }\right\}=\{2.5,-1.5,0.5\}$

\subsection{Macroscopic objective optimization}

For MACOO based on the squeeze-film problem, the influence of the temporal variations in the macroscopic response will be captured by constructing the objective function based on the macroscopic interface dissipation (47) integrated over a cycle, ie,

$$
\varphi=\int_{T} \Pi \mathrm{d} t=\underbrace{\int_{T} \int_{\mathcal{L}} \boldsymbol{G} \cdot \boldsymbol{A} \boldsymbol{G} \mathrm{d} a \mathrm{~d} t}_{\varphi^{4}}+\underbrace{\overline{\boldsymbol{U}} \cdot\left(|\mathcal{L}| \int_{T} \boldsymbol{F} \mathrm{d} t\right) \overline{\boldsymbol{U}}}_{\varphi^{F}} .
$$


Due to the sinusoidal profile for $\frac{\partial h_{0}}{\partial t}$, it is sufficient to compute the time integral over half a cycle.

In the classical setup, the second contribution in (60) vanishes because $\overline{\boldsymbol{U}}=\mathbf{0}$. For this case, Figure 9 presents a series of micro-textures, which span the range from a maximum dissipation to a homogeneous surface response. The corresponding evolution in the surface topology as well as the change in the macroscopic response of the micro-texture designs are further summarized in Figure 10. Similar to the earlier MiCOO study of Figure 8, the flexible optimization framework is able to deliver sharp or smooth topologies depending on the objective function of interest. The macroscopic response is monitored through the dissipation $\Pi$ from (47) and the load capacity $L_{0}$ from (55). Although the objective function is dissipation based, it is observed that a similar ordering for the micro-texture designs is observed for the load capacity as well. In particular, for this problem, the micro-texture that maximizes the dissipation also maximizes the load capacity.

In the modified setup, a tangential velocity $\overline{\boldsymbol{U}}$ is additionally assigned to $\mathcal{P}^{+}$. The orientation of $\overline{\boldsymbol{U}}$ is fixed to an angle of $\pi / 4$ from the vertical axis, whereas its magnitude is gradually increased. The micro-texture designs that maximize the objective function (60) for each case are presented in Figure 11. These designs start with $\|\overline{\boldsymbol{U}}\|=0$, which corresponds to the dissipation maximizing result from Figure 9 with $\varphi=\varphi^{A}$, and gradually approach a limit where $\varphi^{F}$ dominates $\varphi$ as $\|\overline{\boldsymbol{U}}\|$ increases. In order to highlight this limit more clearly, the micro-texture design that maximizes $\varphi^{F}$ alone is additionally displayed and indicated with $\|\overline{\boldsymbol{U}}\| \rightarrow \infty$. Note that the macroscopic response of the micro-texture, as characterized by the flow factor tensors, is isotropic for $\|\overline{\boldsymbol{U}}\|=0$, whereas it becomes increasingly anisotropic with increasing $\|\overline{\boldsymbol{U}}\|$. The nontrivial variation in the optimal micro-texture design topology during this transition is effectively captured through the homogenization-based topology optimization framework.

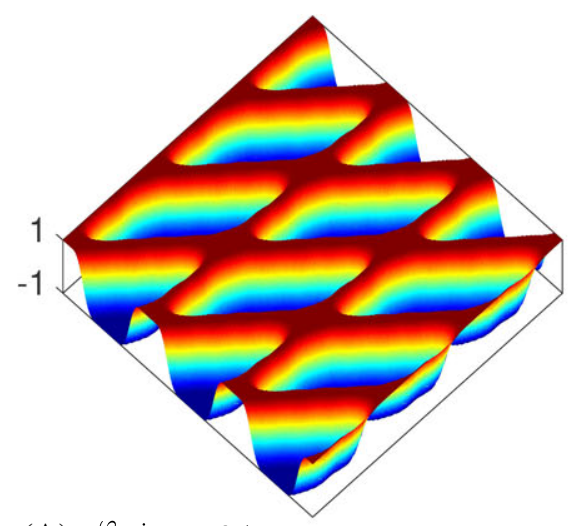

(A) $\frac{\varphi_{\min }}{\varphi_{0}}=0.7$

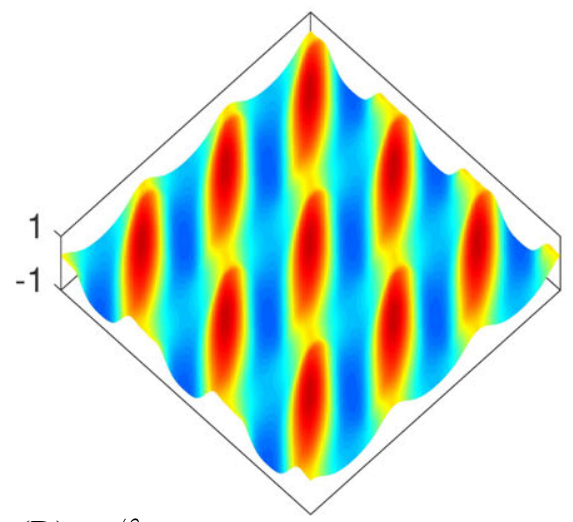

(D) $\frac{\varphi}{\varphi_{0}}=1.1$

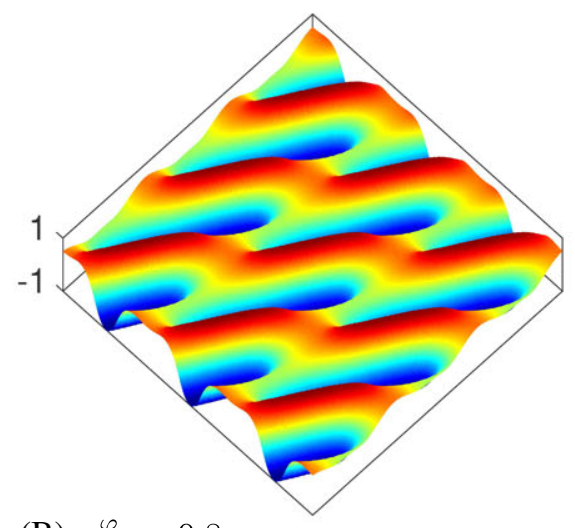

(B) $\frac{\varphi}{\varphi_{0}}=0.8$

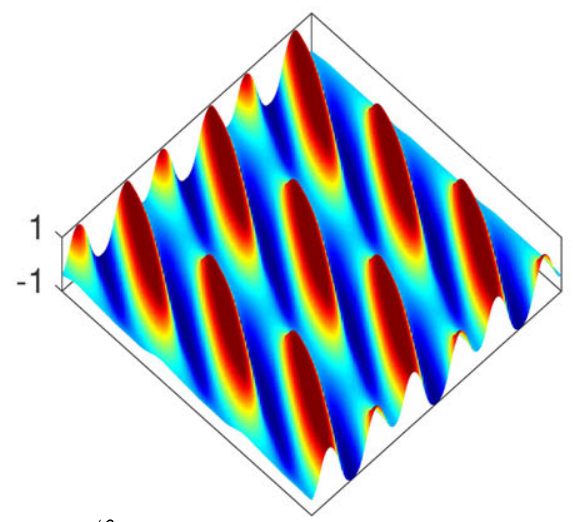

(E) $\frac{\varphi}{\varphi_{0}}=1.3$

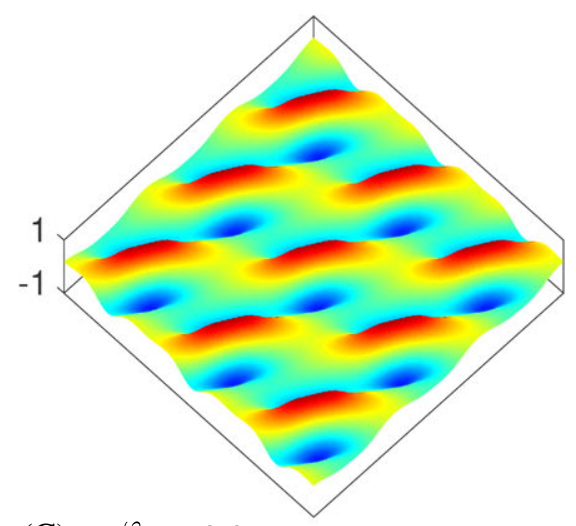

(C) $\frac{\varphi}{\varphi_{0}}=0.9$

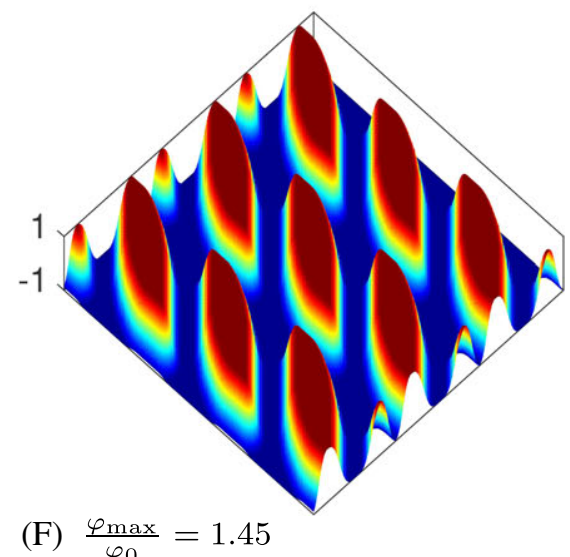

FIGURE 8 For a fixed angle $(\pi / 6)$ for $\boldsymbol{G}$ and $\overline{\boldsymbol{U}}$ with magnitudes $\|\boldsymbol{G}\|=2$ and $\|\overline{\boldsymbol{U}}\|=10, \varphi / \varphi_{0}$ is driven to a value between its maximum and minimum using $\left\{h_{0}, \bar{h}_{\min }, \bar{h}_{\max }\right\}=\{2,-1,1\}$ 


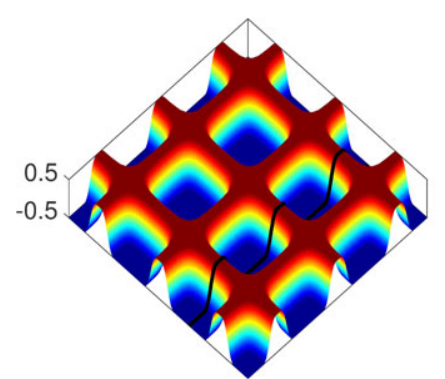

(A.1) $\frac{\varphi_{\max }}{\varphi_{0}}=1.45$

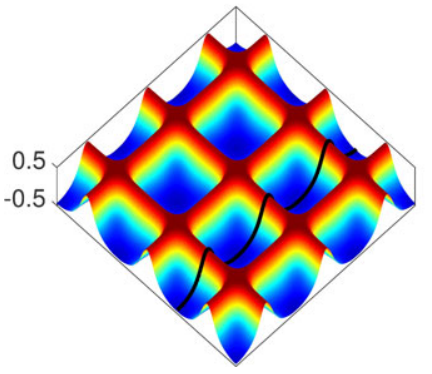

(A.3) $\frac{\varphi}{\varphi_{0}}=1.2$

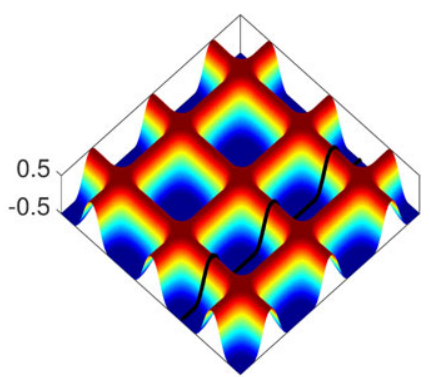

(A.2) $\frac{\varphi}{\varphi_{0}}=1.3$

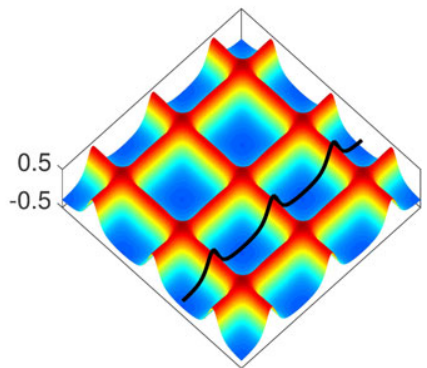

(A.4) $\frac{\varphi}{\varphi_{0}}=1.1$

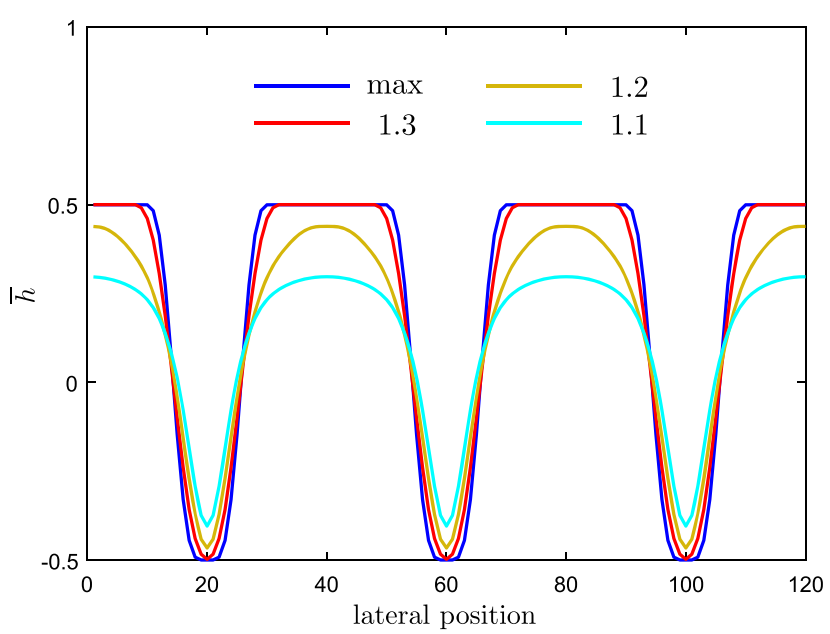

(B) texture profiles

FIGURE 9 (A) For the classical squeeze-film problem, the macroscopic objective optimization (MACOO) objective function $\varphi$ from (60) is first maximized and subsequently also driven to a specified value between the maximum value $\varphi_{\max }$ and the homogeneous surface value $\varphi_{0}$. Here, $\left\{\bar{h}_{\min }, \bar{h}_{\max }\right\}=\{-0.5,0.5\}$. Sample texture profiles along the black lines are compared in (B)

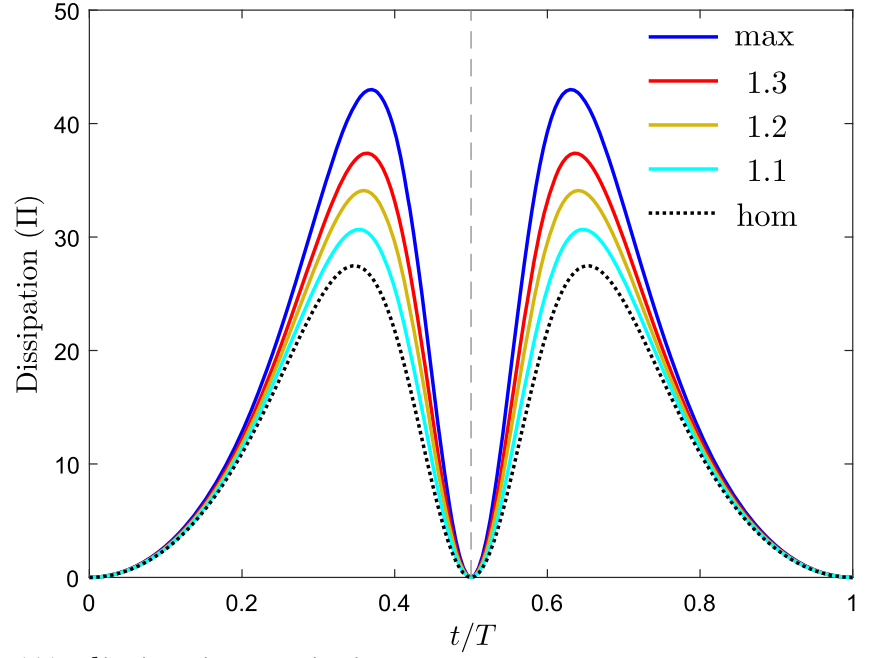

(A) dissipation variation

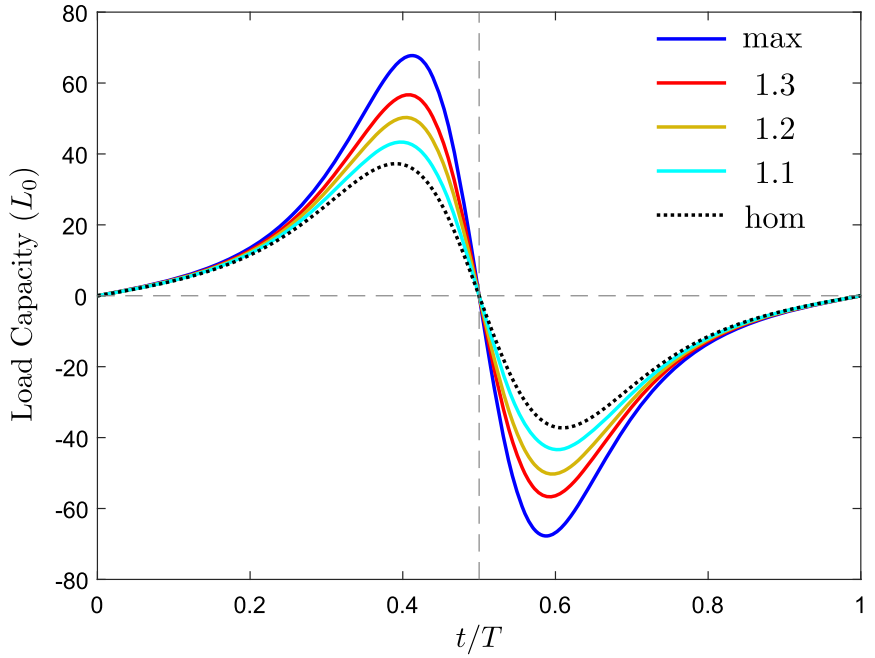

(B) load variation

FIGURE 10 The dissipation and load curves for the micro-textures from Figure 9 are presented. Although the computations are carried out over half a cycle using $N_{T}=10$ time steps, these curves were generated by computing the macroscopic response of the micro-texture design over the whole cycle using 100 times steps for a better representation of the temporal variations. The significant deviation from the homogeneous (hom) surface response highlights the influence of the micro-texture

\section{8 | MICRO-TEXTURE OPTIMALITY}

\section{1 | Global optimality}

The optimization framework summarized in Section 6.1 does not guarantee global optimality. Clearly, various ingredients of the numerical setup (eg, topology filtering operation, finite element discretization, optimization stopping criterion) 


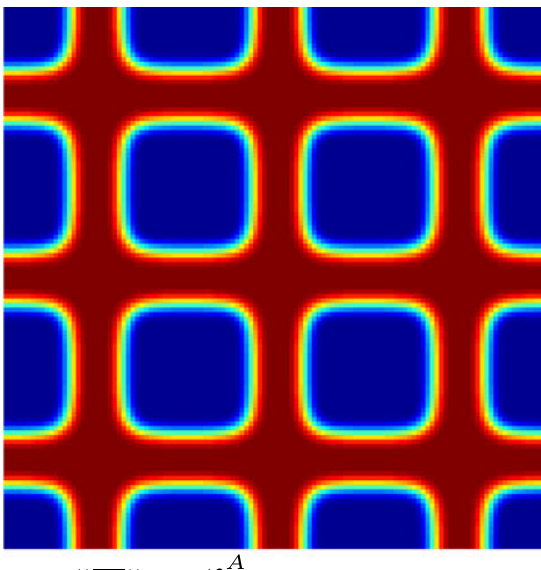

(A) $\|\overline{\boldsymbol{U}}\|=0, \frac{\varphi_{\max }^{A}}{\varphi_{0}^{A}}=1.45$

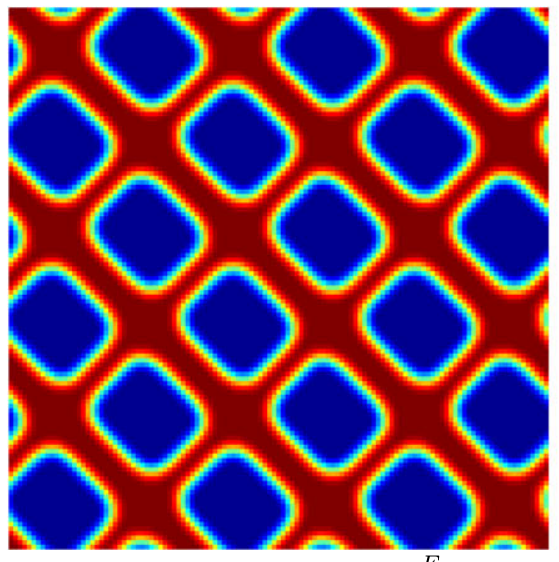

(D) $\|\overline{\boldsymbol{U}}\|=90, \frac{\varphi_{\max }}{\varphi_{0}}=1.26, \frac{\varphi^{F}}{\varphi^{A}}=1.96$

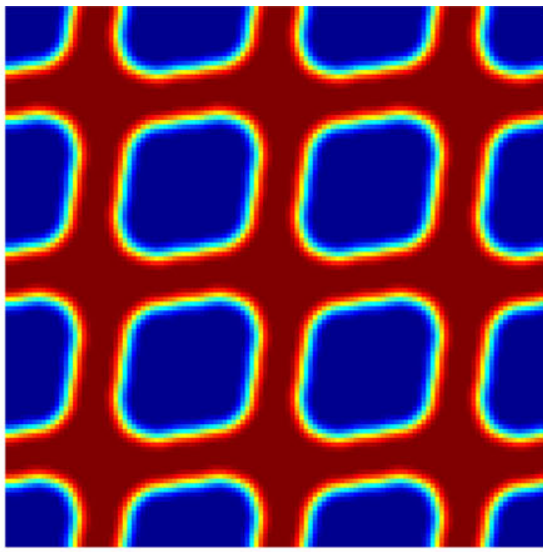

(B) $\|\bar{U}\|=30, \frac{\varphi_{\max }}{\varphi_{0}}=1.40, \frac{\varphi^{F}}{\varphi^{A}}=0.20$

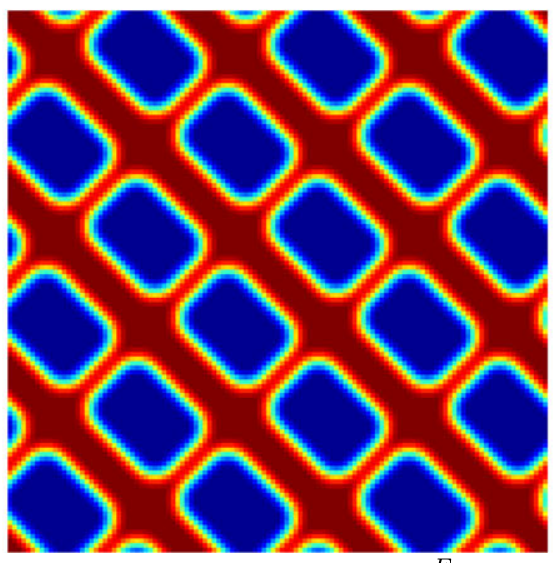

(E) $\|\overline{\boldsymbol{U}}\|=120, \frac{\varphi_{\max }}{\varphi_{0}}=1.25, \frac{\varphi^{F}}{\varphi^{A}}=5.17$

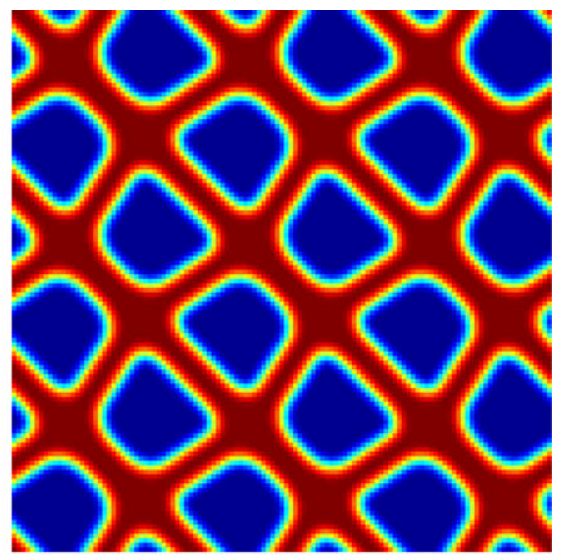

(C) $\|\bar{U}\|=50, \frac{\varphi_{\max }}{\varphi_{0}}=1.3, \frac{\varphi^{F}}{\varphi^{A}}=0.59$

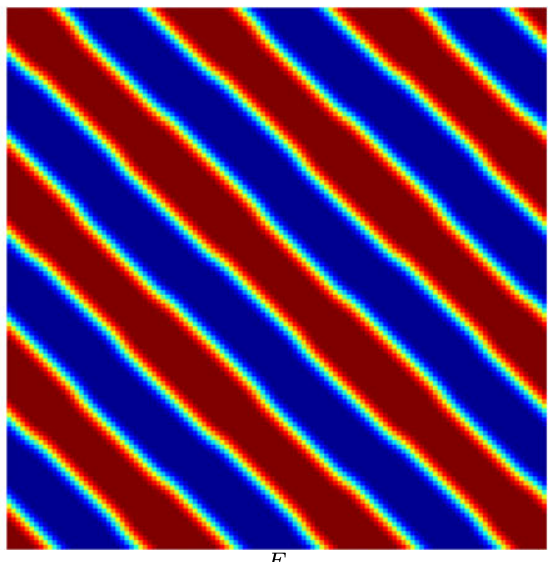

(F) $\|\overline{\boldsymbol{U}}\| \rightarrow \infty, \frac{\varphi_{\max }^{F}}{\varphi_{0}^{F}}=1.28$

FIGURE 11 For the modified squeeze-film problem with a fixed orientation of $\overline{\boldsymbol{U}}$ at an angle of $\pi / 4$ from the vertical axis, the total dissipation as characterized by the macroscopic objective optimization (MACOO) objective function $\varphi$ from (60) is maximized for a range of $\|\overline{\boldsymbol{U}}\|$ values. Here, $\left\{\bar{h}_{\min }, \bar{h}_{\max }\right\}=\{-0.5,0.5\}$. Although the limit where $\varphi^{F}$ dominates $\varphi$ is indicated with $\|\overline{\boldsymbol{U}}\| \rightarrow \infty$, this limit is practically achieved already beyond $\|\overline{\boldsymbol{U}}\|=130$

will already influence the degree to which the micro-texture design is optimal. However, apart from this influence, there may be other factors that govern the qualitative or quantitative aspects of optimality such as, respectively, how well the design reproduces the features of the theoretically optimal topology or how close its objective function value is to the theoretical optimum. The purpose of this section is to critically examine the results presented in Section 7 with respect to micro-texture optimality.

Upon closer examination, Figure 3 reveals that some of the micro-texture designs may be suboptimal. This shortcoming is foremost qualitative in nature. More specifically, the optimal micro-texture has grooves for $\theta=\{0, \pi / 4, \pi / 2\}$ but not for intermediate angles. One would expect that, if a design with grooves is optimal, then its rotation to an intermediate angle should also be optimal. To highlight this point, the normalized objective function (NOF) value

$$
\eta=\varphi / \varphi_{o}
$$

that was monitored in these examples will be examined for rotated designs. Now, the NOF value corresponding to the micro-texture design at a given $\theta$ that sets the direction for the macroscopic vectors $\boldsymbol{G}$ and $\overline{\boldsymbol{U}}$ will be indicated by $\tilde{\eta}(\theta)$. For all $\theta$ values, the homogeneous surface NOF value is $\eta_{0}=1$. Furthermore, let $\boldsymbol{A}(0)$ and $\boldsymbol{F}(0)$ denote, respectively, the macroscopic flow and stress factor tensors corresponding to a micro-texture design for $\theta=0$, which has grooves in all three optimization cases of Figure 3. If this design is rotated clockwise through an angle $\alpha$, the macroscopic response of the rotated design can be characterized through $\boldsymbol{A}(\alpha)=\boldsymbol{Q} \boldsymbol{A}(0) \boldsymbol{Q}^{T}$ and $\boldsymbol{F}(\alpha)=\boldsymbol{Q F}(0) \boldsymbol{Q}^{T}$, where $\boldsymbol{Q}(\alpha)$ is the corresponding 


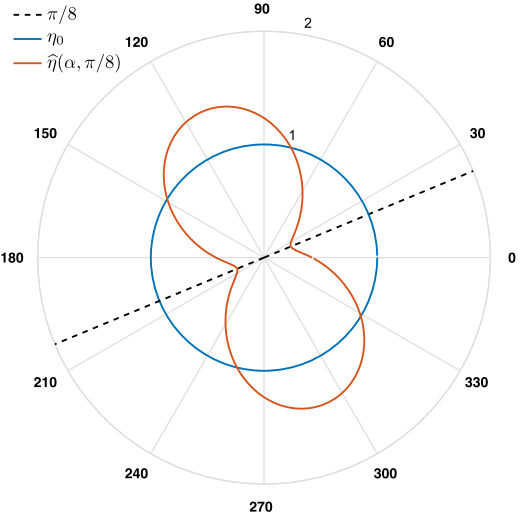

(A) case of Figure $3(\mathrm{a}-2): \varphi^{A} \rightarrow \min$

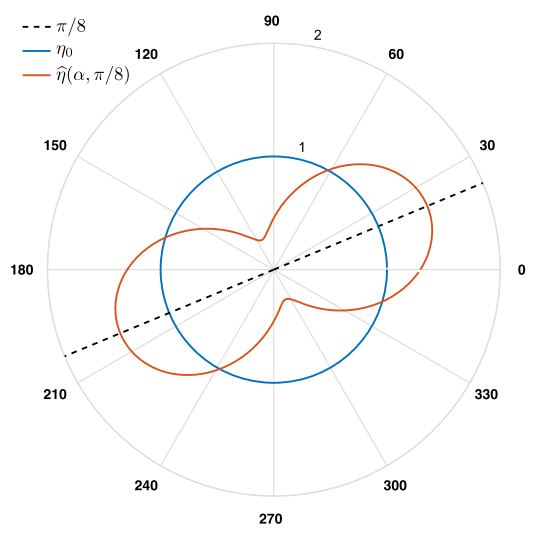

(B) case of Figure 3(b-2): $\varphi^{A} \rightarrow \max$

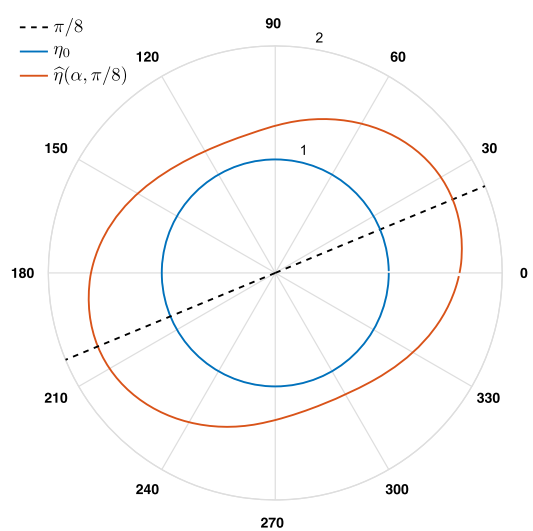

(C) case of Figure $3(\mathrm{c}-2): \varphi^{F} \rightarrow \max$

FIGURE 12 The $\theta=\pi / 8$ cases of Figure 3 are revisited by evaluating the normalized objective function value $\hat{\eta}(\alpha, \pi / 8)$ based on the micro-texture design for $\theta=0$ that is rotated clockwise through an angle $\alpha=0 \rightarrow 2 \pi$, following the discussion in Section 8.1. The best performance is observed when $\alpha=\theta$, with $\hat{\eta}(\pi / 8, \pi / 8)=\tilde{\eta}(0)$

rotation tensor in two dimensions. Now, $\boldsymbol{A}(\alpha)$ and $\boldsymbol{F}(\alpha)$ may be employed in the evaluation of the NOF value at a given $\theta$, delivering an NOF value, which will be indicated by $\hat{\eta}(\alpha, \theta)$. These values are plotted in Figure 12 for $\theta=\pi / 8$, where one observes that the best performance of the rotated design occurs when $\alpha=\theta$, ie, when the grooves are perpendicular or parallel to the direction of the macroscopic vectors depending on the particular case of interest, with the corresponding NOF value $\hat{\eta}(\theta, \theta)=\tilde{\eta}(0)$. This observation, by itself, would clearly not be a proof of the optimality of the rotated design in general. However, in view of the expectation stated earlier, the best performance also corresponds to the optimal one, to within an error that is associated with the possible suboptimality of the values for $\boldsymbol{A}(0)$ and $\boldsymbol{F}(0)$. Indeed, it can now also be observed that the corresponding NOF values $\tilde{\eta}(\pi / 8)$ in Figure 3 are suboptimal with respect to the rotated design performance $\hat{\eta}(\pi / 8, \pi / 8)$. Hence, despite the possible lack of uniqueness in these types of topology optimization problems, the lack of groove formation is presently an indication of suboptimality.

On the other hand, the shortcoming mentioned earlier does not appear to be as significant from a quantitative perspective if the influence of the micro-texture is assessed with respect to the homogeneous surface response. Indeed, the difference between the NOF values corresponding to different $\theta$ values in Figure 3 is generally in the order of $\mathcal{O}\left(10^{-2}-10^{-1}\right)$, whereas the benefit gained by introducing the micro-texture is in the order of $\mathcal{O}\left(10^{-1}-1\right)$, almost an order of magnitude larger. Moreover, as a specific example, it follows that the relative suboptimality of the designs for $\theta=\pi / 8$ with respect to $\hat{\eta}(\pi / 8, \pi / 8)=\tilde{\eta}(0)$ is also of similar magnitude. Note that, primarily, due to the interaction of groove orientation with numerical discretization, there is already a small mismatch between the NOF values $\tilde{\eta}(0)$ and $\tilde{\eta}(\pi / 4)$, both of which are associated with the expected groove formation but the influence of discretization on the diagonal formation appears to be more pronounced in view of its relative suboptimality. Compared to these, the NOF values for an intermediate angle such as $\theta=\pi / 8$ are consistently larger/smaller for minimization/maximization studies but the difference is comparable to the mismatch between the cases with groove formation. In other words, despite the qualitative variation of the micro-texture topology from the expected one, its quantitative response is very close, the error being much smaller than the overall influence of the micro-texture.

The purpose of the remaining discussion is to probe deeper into this observation, examine its underlying causes, further assess the degree of suboptimality, and finally propose a remedy to either alleviate or eliminate it.

\section{2 | Analytical estimates}

In the first step, toward the interpretation of the results in Figure 3, which form the basis of the present discussion, analytical estimates for micro-textures with grooves will be employed. Although the purpose here is neither to prove that such textures are optimal nor to seek ones that are to derive relevant analytical estimates, the macroscopic flow and stress factor tensors can easily be quantified analytically for such special topologies. 


\subsection{1 | Flow factor tensor}

The mechanical role of the flow factor tensor $\boldsymbol{A}$ is similar to the thermal conductivity tensor in a two-dimensional setting for which there are well-established estimates and bounds. ${ }^{65}$ For a micro-texture with grooves, $\boldsymbol{A}$ is diagonal with components

$$
A_{\perp}=\left\langle a^{-1}\right\rangle^{-1} \quad, \quad A_{\|}=\langle a\rangle,
$$

where $\|(\perp)$ indicates the direction that is parallel (perpendicular) to the grooves. Denoting the analytical estimates for NOF values via $\bar{\eta}$, one then concludes

$$
\bar{\eta}_{\min }^{A}=A_{\perp} / a_{0} \leq 1 \quad, \quad \bar{\eta}_{\max }^{A}=A_{\|} / a_{0} \geq 1 .
$$

The reader is referred to the works of Almqvist et $\mathrm{al}^{66}$ and Lukkassen et $\mathrm{al}^{67}$ for an analysis of bounds associated with the flow factor tensors.

In applying these results to Figure 3, it will be assumed that the transition between the minimum and maximum values of $\bar{h}$ is sharp, ie, $\rho$ picks a value of either 0 or 1. Then, cases (a) and (b) have an area fraction equal to 0.6 for $\bar{h}_{\min }$ and the value for case (c) is 0.5. Accordingly, also making use of the results from Figure 12, for Figure 3(a-2), one obtains

$$
\left\{\bar{\eta}_{\text {min }}^{A}=0.222\right\}<\left\{\hat{\eta}_{\text {min }}^{A}(\pi / 8, \pi / 8)=0.256\right\}<\left\{\tilde{\eta}_{\text {min }}^{A}(\pi / 8)=0.326\right\} \text {, }
$$

and, for Figure 3(b-2), one obtains

$$
\left\{\bar{\eta}_{\max }^{A}=1.559\right\}>\left\{\hat{\eta}_{\max }^{A}(\pi / 8, \pi / 8)=1.468\right\}>\left\{\tilde{\eta}_{\max }^{A}(\pi / 8)=1.343\right\}
$$

These results indicate that it is not appropriate to directly compare the design performance $\tilde{\eta}(\theta)$ directly with the analytical estimate $\bar{\eta}$ because even the rotated micro-texture performance $\hat{\eta}(\theta, \theta)$ does not match it, ie, $\boldsymbol{A}(0)$ is suboptimal. Note that the major source of this latter mismatch is the fact that a linear filter was chosen in Section 6.1 for topology optimization, which leads to smoothly varying values of $\rho$ between 0 in 1 in certain portions of the domain, whereas the analytical values are based on a sharp transition. In other words, the suboptimality associated with the lack of groove formation is better judged when compared to the performance of the rotated design for a given set of numerical parameters, which determine the design space.

\subsection{2 | Stress factor tensor}

A similar analysis may be carried out for the stress factor tensor $\boldsymbol{F}$ that is also diagonal for such a special micro-texture with components $F_{\perp}$ and $F_{\|}$, which, although straightforward, appears not to have been attempted in the literature. For this purpose, consider the setting where the flow is perpendicular to the grooves with the condition $\|\overline{\boldsymbol{U}}\|=1$, similar to the case of Figure 3(c-1). Following the discussion in Section 3.1 in the context of Section 4 and simplifying the notation by dropping subscript $\varepsilon$ on the microscopic vectors in the limit $\varepsilon \rightarrow 0$, the nonzero microscopic fluid flux component must be constant across the domain in this case with a value $Q_{\perp}=\left\langle q_{\perp}\right\rangle=q_{\perp}=-a g_{\perp}+b$, where $g_{\perp}$ is the nonzero component of the microscopic pressure gradient, which necessarily varies across the texture according to $g_{\perp}=-a^{-1}\left(Q_{\perp}-b\right)$. Now, in order to obtain a flow setting that is governed only by the tangential velocity macroscopically, the condition $G_{\perp}=\left\langle g_{\perp}\right\rangle=0$ will be imposed, leading to the result $Q_{\perp}=\left\langle a^{-1} b\right\rangle\left\langle a^{-1}\right\rangle^{-1}$ and hence to $g_{\perp}=-a^{-1}\left(\left\langle a^{-1} b\right\rangle\left\langle a^{-1}\right\rangle^{-1}-b\right)$. Recalling the discussion of Section 3.2 in the context of (9) and (36), this result may be employed in the microscopic tangential traction expression $\tau_{\perp}^{+}=\tau_{\nu \perp}^{+}=b g_{\perp}+e$, leading to an expression for $\left\langle\tau_{\perp}^{+}\right\rangle=\overline{\mathcal{T}}_{\perp}=F_{\perp}$. The corresponding analysis for the case when the flow is parallel to the grooves is straightforward because $g_{\|}=0$, directly leading to $\tau_{\|}^{+}=\tau_{\nu \|}^{+}=e$ and hence to an expression for $\left\langle\tau_{\|}^{+}\right\rangle=\overline{\mathcal{T}}_{\|}=F_{\|}$. The explicit results for these diagonal components are

$$
F_{\|}=\left\langle e^{-1}\right\rangle^{-1} \quad, \quad F_{\perp}=-\left\langle a^{-1} b\right\rangle^{2}\left\langle a^{-1}\right\rangle^{-1}+\left\langle a^{-1} b^{2}\right\rangle+\langle e\rangle .
$$

Note that $\left\langle e^{-1}\right\rangle^{-1}=e_{0}$ and $F_{\perp} \geq\langle e\rangle$. The latter inequality follows from the fact that $(3 \mu)^{-1}\left(F_{\perp}-\langle e\rangle\right)=-\left\langle h^{-2}\right\rangle^{2}\left\langle h^{-3}\right\rangle^{-1}+$ $\left\langle h^{-1}\right\rangle$ is nonnegative because $\left\langle h^{-2}\right\rangle^{2} \leq\left\langle h^{-1}\right\rangle\left\langle h^{-3}\right\rangle$ as a consequence of the Cauchy-Schwarz inequality. One then concludes

$$
\bar{\eta}_{\min }^{F}=F_{\|} / e_{0}=1 \quad, \quad \bar{\eta}_{\max }^{F}=F_{\perp} / e_{0} \geq 1,
$$

which are in agreement with the analysis from Section 7.2.1. 
In applying these results to Figure 3, it will again be assumed that the transition between the minimum and maximum values of $\bar{h}$ is sharp. Accordingly, also making use of the results from Figure 12, for Figure 3(c-2), one obtains

$$
\left\{\bar{\eta}_{\max }^{F}=1.762\right\}>\left\{\hat{\eta}_{\max }^{F}(\pi / 8, \pi / 8)=1.716\right\}>\left\{\widetilde{\eta}_{\max }^{F}(\pi / 8)=1.652\right\}
$$

The influence of the linear filter is observed again in view of the gap between the rotated micro-texture performance $\hat{\eta}_{\max }^{F}(\pi / 8, \pi / 8)$ and the analytical estimate $\bar{\eta}_{\max }^{F}$, ie, $\boldsymbol{F}(0)$ is suboptimal, which highlights that the design performance $\widetilde{\eta}_{\max }^{F}(\pi / 8)$ should be compared with $\widehat{\eta}_{\max }^{F}(\pi / 8, \pi / 8)$. The gap between these latter two values is observed to be small despite the lack of groove formation. Note that the minimum value of $\hat{\eta}(\alpha, \pi / 8)$ in Figure 12(c) is greater than the analytical minimum, also primarily due to the combined influence of discretization and filtering.

\section{3 | Domain shape}

The preceding discussion assessed the degree of suboptimality for the micro-texture designs. Irrespective of the quantitative nature of this suboptimality, which was observed to be small, an outstanding issue is its qualitative nature that is associated with the lack of groove formation. In order to address this issue, note that the unit-cell domain employed in the optimization of the micro-texture topology was assigned a square shape in Section 7.1. As noted therein, the graphical depiction of a micro-texture design is based on the $3 \times 3$ tiling of the unit-cell topology. Now, it is clear from the results in Figure 3 corresponding to $\theta=0$ that the chosen filter radius allows the formation of only a single groove over a unit-cell. This, however, is a limiting factor for the formation of the optimal micro-texture topology because a square unit-cell should, in general, accommodate more than one groove for intermediate angles to satisfy periodicity. Alternatively stated, in general, a unit-cell with a single groove at an arbitrary angle should not have a square shape. Therefore, one might expect to recover groove formation if the unit-cell is assigned a rectangular shape with the correct aspect ratio. Denoting the horizontal and vertical dimensions of a rectangular unit-cell with $\ell_{m}^{x}$ and $\ell_{m}^{y}$, respectively, the aspect ratio may be defined as $\gamma=\ell_{m}^{y} / \ell_{m}^{x}$. The ideal aspect ratio $\bar{\gamma}$ is one that allows the groove to run along the diagonal of the rectangle. In the context of Figure 3, this ideal value is $(0<\theta<\pi / 2)$

$$
\bar{\gamma}_{\min }^{A}=\tan (\theta), \quad \bar{\gamma}_{\max }^{A}=\cot (\theta), \quad \bar{\gamma}_{\max }^{F}=\tan (\theta) .
$$

In order to demonstrate the influence of the aspect ratio, the setting of Figure 3(a-2) is now revisited in Figure 13 by carrying out optimization with different unit-cell shapes. Note that groove formation is not observed unless $\gamma$ is close to the ideal value $\bar{\gamma}_{\text {min }}^{A}$. Moreover, even when it forms, the corresponding design performance is suboptimal with respect to the one with $\bar{\gamma}_{\mathrm{min}}^{A}$. At this ideal value, the micro-texture design delivers $\eta_{\mathrm{min}}^{A}=0.281$, which, in view of the discussion of Section 8.1, should be compared not with the design performance $\widetilde{\eta}_{\min }^{A}(0)$ of Figure 3(a-1) but rather with $\widetilde{\eta}_{\min }^{A}(\pi / 4)$ of Figure 3(a-3), where a similar diagonal groove formation occurs. Indeed, it is observed that an almost identical performance is obtained in both cases as one might anticipate. Therefore, within the limits posed by the combined influence of discretization and filtering, the design performance may be improved toward the best possible value by altering the unit-cell shape, such as through the aspect ratio. Presently, the ideal shape can easily be determined beforehand. In more complicated cases such as when both $\boldsymbol{A}$ and $\boldsymbol{F}$ contribute to the objective function, it is neither straightforward nor meaningful to determine the ideal shape beforehand because groove formation may not align with $\boldsymbol{G}$ or $\overline{\boldsymbol{U}}$ and, in fact, groove formation may not occur at all as in Section 7.3. In such cases, even if performance improvement is targeted through the variation of the unit-cell shape, the best possible value is already observed to be limited through factors such as discretization and filtering. Therefore, it is desirable to seek an alternative method for improving the design performance in a simpler fashion, possibly toward the analytical estimate when it is available, so that a step may be taken toward ensuring the global optimality of the micro-texture. Such a method is discussed next.

\section{4 | Domain size}

In Section 7.1, it was indicated that the absolute edge length $\ell_{m}$ of the square unit-cell only has relevance with respect to the filter radius $R$. Indicating the default choice for the edge length by $\ell_{o}$, the default radius is such that $R / \ell_{o}=0.1$. If this ratio is preserved and $\ell_{m}$ is increased, the features of the micro-texture will increase in dimension but the overall topology and hence the design performance will be preserved. If, on the other hand, the value of $R$ is preserved and $\ell_{m}$ is increased, then one obtains a setting where the micro-texture features of a given size are allowed to develop over a 


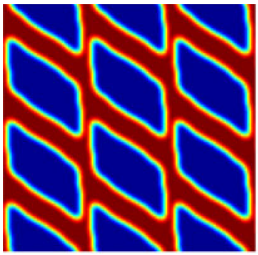

(A.1) $\gamma=0.1$

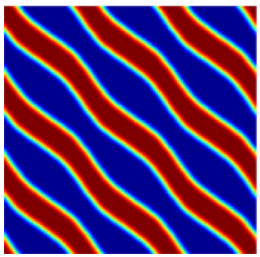

(A.4) $\gamma=0.6$

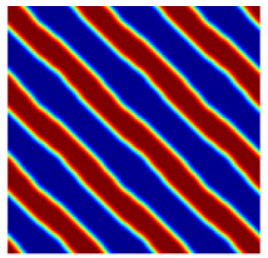

(A.2) $\gamma=0.2$

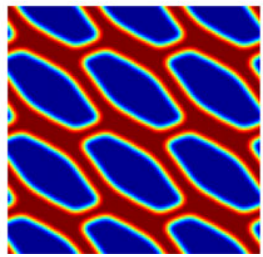

(A.5) $\gamma=0.7$

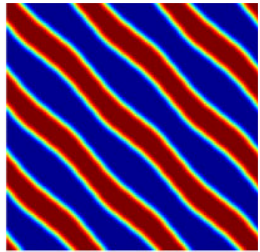

(A.3) $\gamma=\bar{\gamma}_{\min }^{A}$

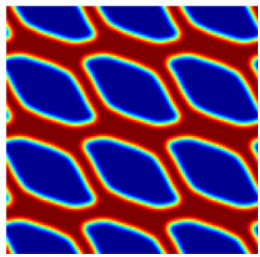

(A.6) $\gamma=0.9$

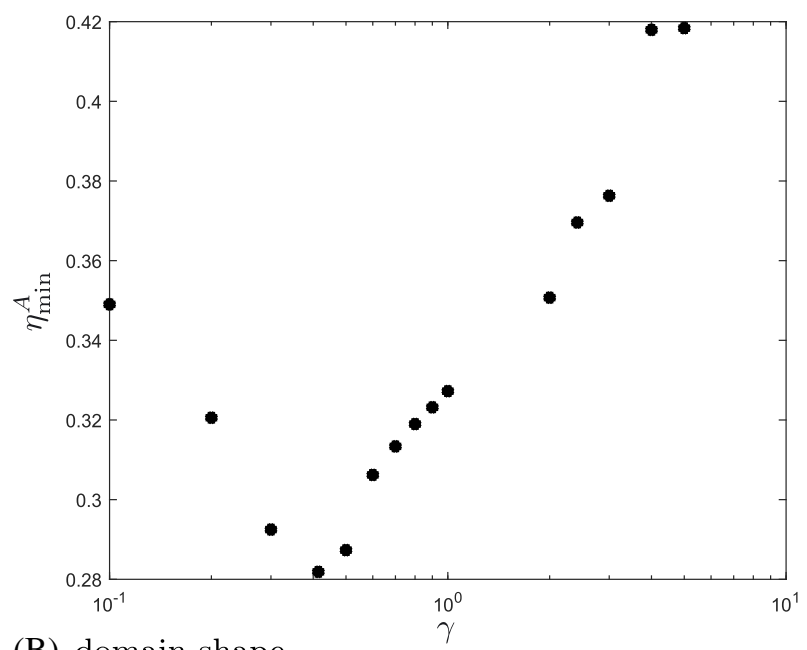

(B) domain shape

FIGURE 13 (A) The setting of Figure 3(a-2) is revisited with unit-cells having different aspect ratios, following the discussion in Section 8.3. The micro-textures are depicted by mapping them onto a square geometry for a simpler visual comparison. (B) The variation of the design performance measure $\eta_{\min }^{A}$ is plotted as a function of the aspect ratio $\gamma$. Figure 3(a-2) employs $\gamma=1$. The minimum value of $0.281 \approx \tilde{\eta}_{\min }^{A}(\pi / 4)$, on the other hand, is observed for the ideal aspect ratio $\bar{\gamma}_{\min }^{A}=\tan (\pi / 8)$

larger domain. Because groove formation is primarily limited by the periodicity constraint on the micro-texture topology near the design domain edges, as remarked in Section 8.3, one may anticipate that this limitation is an edge effect, whose influence may diminish with increasing domain size $\ell_{m}$. This idea is tested in Figure 14 and the expected groove formation is indeed recovered gradually with increasing domain size. Simultaneously, recalling the ordering in (64), the design performance approaches not to $\widetilde{\eta}_{\text {min }}^{A}(\pi / 4)$ of Figure 3(a-3) as earlier observed in Section 8.3 but to a value which lies even below the rotated design performance $\hat{\eta}_{\min }^{A}(\pi / 8, \pi / 8)$ and toward the analytical estimate $\bar{\eta}_{\text {min }}^{A}$, although some gap still remains. This is an indication of the diminishing but nonvanishing combined influence of discretization and filtering. Despite the remaining gap, the improvement in the quantitative and qualitative optimality of the micro-texture design is significant and demonstrates a simple mechanism through which the degree of optimality of a micro-texture design can

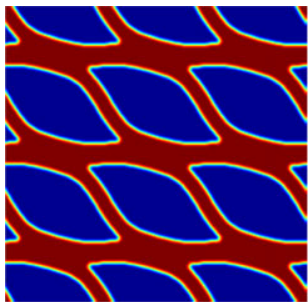

(A.1) $\ell_{m}=2 \ell_{0}$

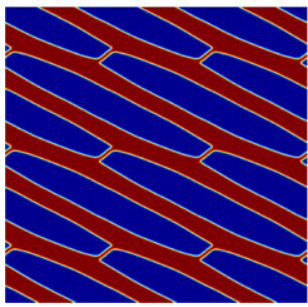

(A.3) $\ell_{m}=4 \ell_{o}$

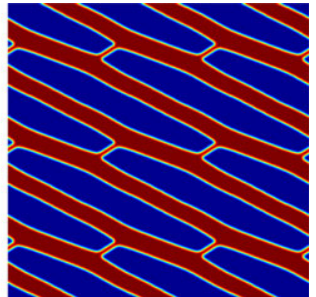

(A.2) $\ell_{m}=3 \ell_{o}$

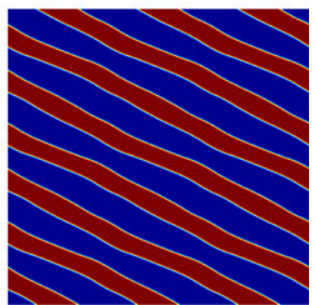

(A.4) $\ell_{m}=5 \ell_{o}$

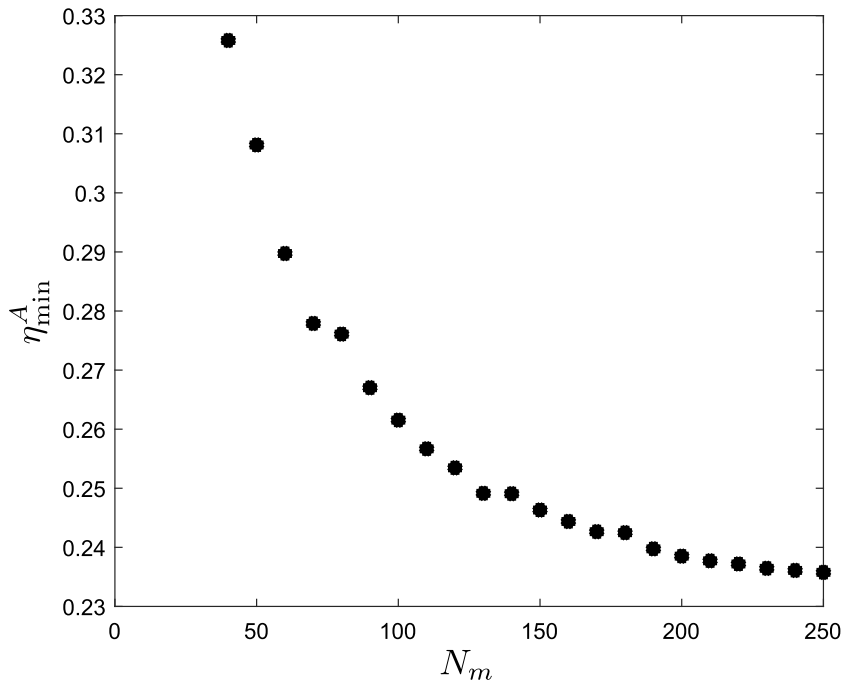

(B) domain size

FIGURE 14 (A) Figure 3(a-2), where $\ell_{m}=\ell_{0}$, is revisited by increasing the design domain size while preserving the filter radius $R$, following the discussion in Section 8.4. The micro-textures are depicted at equal dimensions for a simpler visual comparison. (B) The variation of the design performance measure $\eta_{\min }^{A}$ is plotted as a function of the number of elements $N_{m}$ per domain edge as an alternative measure of size, $N_{m}=40$ corresponding to the domain size of Figure 3(a-2). The normalized objective function (NOF) value nears saturation at $N_{m}=250$ (or, $\ell_{m}=6.25$ ) to a value of $0.236 \approx \bar{\eta}_{\text {min }}^{A}$ 
be assessed without any knowledge of the expected topological features or relevant analytical estimates beforehand. It should, however, again be pointed out that, despite the apparent qualitative shortcomings of some designs, their quantitative performance already demonstrates the benefit of endowing the interface with a micro-texture. Indeed, the degree of optimality in all assessed designs from Section 7 was similar to the examples discussed earlier (not shown). Hence, it can be stated that the numerical setting described in Section 7.1 already delivers results that are indicative of and relevant to the desired tailoring of the microscopic interface for engineering purposes. Moreover, the overall computational framework is not limited in predictive capability with respect to optimality, provided the domain size is verified to be sufficiently large.

\section{9 | CONCLUSION}

The role of the micro-texture in influencing and tuning the macroscopic dissipative response of tribological interfaces is well established. Based on two earlier works, which concentrated on the nondissipative physics of the interface, ${ }^{1,2}$ the goal of this work was to contribute to ongoing efforts in tribology toward the design and optimization of micro-textures in a numerical setting, which can (i) efficiently evaluate the suitability of a given design with respect to optimization objectives that incorporate dissipation, and (ii) subsequently evolve this design within a flexible space of geometries to achieve complex micro-textures that are as close to optimal as possible for a given task or across a class of tasks. Specifically, the first goal was addressed within a thermodynamically consistent two-scale formulation that is based on homogenization and the second goal was addressed by embedding this homogenization approach within a topology optimization framework, which can deliver smoothly as well as sharply varying texture geometries. Various numerical investigations were presented to demonstrate how dissipation influences optimal micro-textures and why it is important to work in a flexible design space. The observed design performance was further critically examined with respect to the quantitative and qualitative aspects of optimality. In particular, the optimization domain geometry was found to effectively act as an additional design variable and a method based on the variation of the domain size was proposed for assessing the degree of optimality. Overall, the results obtained in this study serve to demonstrate the potential advantages of a homogenization-based topology optimization framework for micro-texture design, which is to be compared with alternative approaches that operate within a restricted design space and often through an expensive resolution of the complete mesoscale physics. However, further studies are needed to fully develop the advocated approach toward practical problems of current technological interest. Among these, the ability to address soft micro-textures, which are prone to deformation even at light interface loads and which typically operate under mixed microscopic contact-lubrication conditions, stands out as a particularly challenging task that will be the focus of future research.

\section{ORCID}

İlker Temizer (D) https://orcid.org/0000-0003-3043-7521

Kenjiro Terada (iD https://orcid.org/0000-0001-6799-2233

Junji Kato (1) https://orcid.org/0000-0002-0903-9513

\section{REFERENCES}

1. Waseem A, Temizer İ, Kato J, Terada K. Homogenization-based design of surface textures in hydrodynamic lubrication. Int J Numer Methods Eng. 2016;108:1427-1450.

2. Waseem A, Temizer İ, Kato J, Terada K. Micro-texture design and optimization in hydrodynamic lubrication via two-scale analysis. Struct Multidiscip Optim. 2017;56:227-248.

3. Bendsøe MP, Sigmund O. Topology Optimization: Theory, Methods, and Applications. 2nd ed. Berlin, Germany: Springer-Verlag Berlin Heidelberg; 2004.

4. Christensen PW, Klarbring A. An Introduction to Structural Optimization. Dordrecht, The Netherlands: Springer Netherlands; 2009.

5. Sanchez-Palencia E. Non-Homogeneous Media and Vibration Theory. Berlin, Germany: Springer-Verlag Berlin Heidelberg; 1980.

6. Pavliotis GA, Stuart AM. Multiscale Methods: Averaging and Homogenization. New York, NY: Springer-Verlag New York; 2008.

7. Sigmund O, Torquato S. Composites with extremal thermal expansion coefficients. Appl Phys Lett. 1996;69:3203-3205.

8. Sigmund O. A new class of extremal composites. J Mech Phys Solids. 2000;48:397-428.

9. Chen BC, Silva ECN, Kikuchi N. Advances in computational design and optimization with application to MEMS. Int J Numer Methods Eng. 2001;52(1-2):23-62.

10. Sigmund O. Materials with prescribed constitutive parameters: an inverse homogenization problem. Int J Solids Struct. 1994;31:2313-2329.

11. Neves MM, Rodrigues H, Guedes JM. Optimal design of periodic linear elastic microstructures. Comput Struct. 2000;76:421-429. 
12. Fujii D, Chen BC, Kikuchi N. Composite material design of two-dimensional structures using the homogenization design method. Int $J$ Numer Methods Eng. 2001;50(9):2031-2051.

13. Nakshatrala PB, Tortorelli DA, Nakshatrala KB. Nonlinear structural design using multiscale topology optimization. Part I: static formulation. Comput Methods Appl Mech Eng. 2013;261-262:167-176.

14. Kato J, Yachi D, Terada K, Kyoya T. Topology optimization of micro-structure for composites applying a decoupling multi-scale analysis. Struct Multidisc Optim. 2014;49:595-608.

15. Nishi S, Terada K, Kato J, Nishiwaki S, Izui K. Two-scale topology optimization for composite plates with in-plane periodicity. Int J Numer Methods Eng. 2018;113:1164-1188.

16. Gropper D, Wang L, Harvey TJ. Hydrodynamic lubrication of textured surfaces: a review of modeling techniques and key findings. Tribology International. 2016;94:509-529.

17. Buscaglia GC, Ausas RF, Jai M. Optimization tools in the analysis of micro-textured lubricated devices. Inverse Probl Sci Eng. 2006;14:365-378.

18. Dobrica MB, Fillon M, Pascovici MD, Cicone T. Optimizing surface texture for hydrodynamic lubricated contacts using a mass-conserving numerical approach. Proc Inst Mech Eng J J Eng Tribol. 2010;224(8):737-750.

19. Guzek A, Podsiadlo P, Stachowiak GW. Optimization of textured surface in 2D parallel bearings governed by the Reynolds equation including cavitation and temperature. Tribology Online. 2013;8(1):7-21.

20. Scaraggi M. Optimal textures for increasing the load support in a thrust bearing pad geometry. Tribology Letters. 2014;53(1):127-143.

21. Fesanghary M, Khonsari MM. On the shape optimization of self-adaptive grooves. Tribology Transactions. 2011;54(2):256-264.

22. Shen C, Khonsari MM. Numerical optimization of texture shape for parallel surfaces under unidirectional and bidirectional sliding. Tribology International. 2015;82:1-11.

23. Zhang H, Dong GN, Hua M, Chin KS. Improvement of tribological behaviors by optimizing concave texture shape under reciprocating sliding motion. J Tribol. 2017;139:011702.

24. Hamrock B, Schmid S, Jacobson B. Fundamentals of Fluid Film Lubrication. Boca Raton, FL: CRC Press; 2004.

25. Szeri AZ. Fluid Film Lubrication. New York, NY: Cambridge University Press; 2011.

26. Bayada G, Chambat M. The transition between the Stokes equations and the Reynolds equation: a mathematical proof. Appl Math Optim. 1986;14:73-93.

27. Temizer İ, Stupkiewicz S. Formulation of the Reynolds equation on a time-dependent lubrication surface. Proc Royal Soc A Math Phys Eng Sci. 2016;472:20160032.

28. Yıldıran İN, Temizer İ, Çetin B. Homogenization in hydrodynamic lubrication: microscopic regimes and re-entrant textures. $J$ Tribol. 2017;140:011701.

29. Cope WF. The hydrodynamical theory of film lubrication. Proc R Soc Lond A Math Phys Sci. 1949;197:201-217.

30. Charnes A, Osterle F, Saibel E. On the energy equation for fluid-film lubrication. Proc R Soc Lond A Math Phys Sci. 1952;214:133-136.

31. Bayada G, Chambat M. New models in the theory of the hydrodynamic lubrication of rough surfaces. J Tribol. 1988;110:402-407.

32. Bayada G, Ciuperca I, Jai M. Homogenized elliptic equations and variational inequalities with oscillating parameters. Application to the study of thin flow behavior with rough surfaces. Nonlinear Anal Real World Appl. 2006;7:950-966.

33. Tripp JH. Surface roughness effects in hydrodynamic lubrication: the flow factor method. J Lubr Technol. 1983;105:458-463.

34. Prat M, Plouraboué F, Letalleur N. Averaged Reynolds equation for flows between rough surfaces in sliding motion. Transp Porous Media. 2002;48:291-313.

35. Patir N, Cheng HS. An average flow model for determining effects of three-dimensional roughness on partial hydrodynamic lubrication. J Lubr Technol. 1978;100:12-17.

36. Elrod HG. Thin-film lubrication theory for Newtonian fluids with surface possessing striated roughness or grooving. $J$ Lubr Technol. 1973;95:484-489.

37. Elrod HG. A Review of Theories for the Fluid Dynamic Effects of Roughness on Laminar Lubricating Films. New York, NY: Lubrication Research Laboratory, Columbia University; 1977.

38. Mitsuya Y, Fukui S. Stokes roughness effects on hydrodynamic lubrication. Part I — comparison between incompressible and compressible lubricating films. J Tribol. 1986;108:151-158.

39. Fabricius J, Tsandzana A, Perez-Rafols F, Wall P. A comparison of the roughness regimes in hydrodynamic lubrication. $J$ Tribol. 2017;139:051702.

40. Benhaboucha N, Chambat M, Ciuperca I. Asymptotic behaviour of pressure and stresses in a thin film flow with a rough boundary. Quart Appl Math. 2005;63(2):369-400.

41. Patir N, Cheng HS. Application of average flow model to lubrication between rough sliding surfaces. J Lubr Technol. 1979;101:220-229.

42. Elrod HG. A general theory for laminar lubrication with Reynolds roughness. J Lubr Technol. 1979;101:8-14.

43. Sahlin F, Larsson R, Almqvist A, Lugt PM, Marklund P. A mixed lubrication model incorporating measured surface topography. Part 1: theory of flow factors. Proc Inst Mech Eng J J Eng Tribol. 2009;224:335-351.

44. Buscaglia GC, Ciuperca I, Jai M. The effect of periodic textures on the static characteristics of thrust bearings. J Tribol. 2005;127:899-902.

45. Lukkassen D, Meidell A, Wall P. Analysis of the effects of rough surfaces in compressible thin flow by homogenization. Int J Eng Sci. 2011;49:369-377.

46. Yand B, Laursen T. A mortar-finite element approach to lubricated contact problems. Comput Methods Appl Mech Eng. 2009;198:3656-3669. 
47. Lengiewicz J, Wichrowski M, Stupkiewicz S. Mixed formulation and finite element treatment of the mass-conserving cavitation model. Tribology International. 2014;72:143-155.

48. Ager C, Schott B, Vuong AT, Popp A, Wall WA. A consistent approach for fluid-structure-contact interaction based on a porous flow model for rough surface contact. Int J Numer Methods Eng. 2019. https://doi.org/10.1002/nme.6094. In press.

49. Persson BNJ. Fluid dynamics at the interface between contacting elastic solids with randomly rough surfaces. $J$ Phys Condens Matter. 2010;22:265004.

50. Persson BNJ, Scaraggi M. Lubricated sliding dynamics: flow factors and Stribeck curve. Eur Phys J E. 2011;34:113.

51. Scaraggi M, Carbone G, Persson BNJ, Dini D. Lubrication in soft rough contacts: a novel homogenized approach. Part I - theory. Soft Matter. 2011;7:10395-10406.

52. Scaraggi M, Angerhausen J, Dorogin L, Murrenhoff H, Persson BNJ. Influence of anisotropic surface roughness on lubricated rubber friction: extended theory and an application to hydraulic seals. Wear. 2018;410-411:43-62.

53. Scaraggi M. The friction of sliding wet textured surfaces: the Bruggeman effective medium approach revisited. Proc R Soc Lond A Math Phys Sci. 2015;471:20140739.

54. Kabacaoğlu G, Temizer İ. Homogenization of soft interfaces in time-dependent hydrodynamic lubrication. Computational Mechanics. 2015;56:421-441.

55. Díaz A, Sigmund O. Checkerboard patterns in layout optimization. Structural Optimization. 1995;10(1):40-45.

56. Sigmund O, Petersson J. Numerical instabilities in topology optimization: a survey on procedures dealing with checkerboards, mesh-dependencies and local minima. Structural Optimization. 1998;16(1):68-75.

57. Sigmund O. Morphology-based black and white filters for topology optimization. Struct Multidisc Optim. 2007;33:401-424.

58. Svanberg K, Svärd H. Density filters for topology optimization based on the Pythagorean means. Struct Multidisc Optim. 2013;48:859-875.

59. Bourdin B. Filters in topology optimization. Int J Numer Methods Eng. 2001;50(9):2143-2158.

60. Bruns TE, Tortorelli DA. Topology optimization of non-linear elastic structures and compliant mechanisms. Comput Methods Appl Mech Eng. 2001;190:3443-3459.

61. Bendsøe MP. Optimal shape design as a material distribution problem. Structural Optimization. 1989;1(4):193-202.

62. Rozvany G, Zhou M, Birker T. Generalized shape optimization without homogenization. Structural Optimization. 1992;4:250-252.

63. Svanberg K. The method of moving asymptotes - a new method for structural optimization. Int J Numer Methods Eng. 1987;24:359-373.

64. Buscaglia G, Jai M. Sensitivity analysis and Taylor expansions in numerical homogenization problems. Numerische Mathematik. 2000;85:49-75.

65. Torquato S. Random Heterogeneous Materials: Microstructure and Macroscopic Properties. New York, NY: Springer-Verlag New York; 2002.

66. Almqvist A, Lukkassen D, Meidell A, Wall P. New concepts of homogenization applied in rough surface hydrodynamic lubrication. Int J Eng Sci. 2007;45:139-154.

67. Lukkassen D, Meidell A, Wall P. Homogenization of some variational problems connected to the theory of lubrication. Int J Eng Sci. 2009;47:153-162.

How to cite this article: Çakal BA, Temizer İ, Terada K, Kato J. Microscopic design and optimization of hydrodynamically lubricated dissipative interfaces. Int J Numer Methods Eng. 2019;120:153-178. https://doi.org/10.1002/nme.6129 